\title{
Transient wall pressures in an overexpanded and large area-ratio nozzle
}

\author{
W. J. Baars · C. E. Tinney
}

Received: date / Accepted: date

\begin{abstract}
Surveys of the fluctuating wall pressure were conducted on a sub-scale parabolic-contour rocket nozzle to infer an understanding of the flow and shock structure pattern during fixed and transient operations of the nozzle. During start-up, the nozzle is highly overexpanded, which results in unsteady wall pressure signatures driven by shock foot unsteadiness. Wall pressure data is first analyzed using spatial Fourier transformations to extract the azimuthal modes during various operating states. A time-frequency analysis of the temporal azimuthal mode coefficients is then used to characterize the time-dependent spectral behavior of the wall pressure signatures during start-up. For both fixed and transient operations of the nozzle, the axisymmetric breathing mode $(m=0)$ comprises most of the resolved energy. As for the transient operations alone, slight deviations in ramp rate are shown to considerably influence the amount of unsteadiness that the nozzle wall is exposed to, even though the general spectral and temporal features remain similar. In particular, increased ramp rate resulted in increased wall pressure intensity. Secondly, three major low-frequency events $(f \lesssim 400 \mathrm{~Hz})$ were observed during start-up and are attributed to: (1) FSS $\rightarrow$ RSS transition, (2) the passing of the reattachment line from the first separation bub-
\end{abstract}

\footnotetext{
W. J. Baars $(\bowtie)$

Department of Aerospace Engineering and Engineering Mechanics, The University of Texas at Austin, 1 University Station, C0600, Austin, TX 78712, USA

Tel.: +1 (512) 739-3048

E-mail: baars@utexas.edu

C. E. Tinney

Department of Aerospace Engineering and Engineering Mechanics, The University of Texas at Austin, 1 University Station, C0600, Austin, TX 78712, USA
}

ble, and (3) the 'end-effects regime'. The last of these refers to a condition where a trapped separation bubble opens intermittently to ambient at the nozzle lip.

Keywords First keyword · Second keyword · More

\section{Introduction}

There has been a widespread desire to investigate features associated with Shock Wave Boundary Layer Interactions (SWBLI) in high area-ratio rocket nozzles, since these interactions are responsible for acoustic, vibroacoustic, thermal, and mechanical induced loads that act on the structure and its surroundings. Studies date back to the 1960's with the pioneering work of Arens and Spiegler (1963) and continued on through later years with Nave and Coffey (1973); Schmucker (1973). During the transient operation of a Thrust Optimized Parabolic (TOP) contoured nozzle, the internal flow evolves through several distinctly different flow topographies which depend greatly on the Nozzle Pressure Ratio (NPR) - plenum pressure over ambient pressureand nozzle geometry. These unique flow states have a profound impact on the loads acting on the surface of the nozzle which can vary greatly depending on the timescale of the transient. A typical approach for developing a statistical spectral characterization of the transient nozzle flow is to acquire long data sets at a sequence of fixed NPR conditions along the start-up period (Nguyen et al, 2003; Verma et al, 2006; Baars et al, 2012a). To date, there has been no investigation to determine whether such an approach is satisfactory.

In this article we present results from an experimental study of a sub-scale TOP nozzle during transient operating conditions (start-up). Time-resolved wall pressure measurements are used to develop a physical un- 
derstanding of: (1) the azimuthal behavior of the internal flow during a sequence of start-up conditions as opposed to fixed nozzle pressure ratios, and (2) the socalled 'end-breathing effect' which is believed to cause the most energetic off-axis loads during start-up (Ruf et al, 2009). Before doing so, the accepted model of an internal TOP nozzle flow, in a quasi-steady and axisymmetric sense, is concisely reviewed to highlight the features that are relevant to the current study. These quasi-steady models are inferred from static and dynamic wall pressure measurements and internal flow simulations during fixed NPR conditions (Baars et al, 2012a) and are similar to the ones presented by Frey and Hagemann (2000); Hagemann et al (2002); Nguyen et al (2003); Verma (2009); Ruf et al (2009).

At the initial stage of start-up when NPRs are low, the flow resides in a Free Shock Separation (FSS) state, as shown in Fig. 1a. A single incipient separation of the flow along the interior surface of the nozzle is triggered by an adverse pressure gradient between the region of isentropic expansion and subsonic entrainment. The shock that originates from the incipient separation line interacts with a reflected shock; this reflected shock emanates from the triple-point, which is the location where the Mach disk, internal and reflected shocks coincide. A separated region forms which encompasses a series of compression and expansion waves. This is identified as the 'detached supersonic plume' in Fig. 1a. This separated flow fails to reattach to the nozzle wall at low NPRs due to the lack of outward radial momentum flow induced by the reflected shock; the flow continues as a free supersonic annular jet until it eventually diffuses downstream. Between the detached supersonic plume and the nozzle wall, a recirculating subsonic region forms which entrains ambient air along the nozzle wall.

As the NPR continues to increase, an abrupt transition from a FSS state to a Restricted Shock Separation (RSS) state occurs in the TOP nozzle. More outward radial momentum flow is generated by the reflected shock moving aft during start-up and results in the reattachment of the supersonic plume as is visualized in Fig. 1b. The incipient separation line shifts downstream and aft of the Mach disk. An enclosed region of separated flow forms between the separation shock and an expansion fan reflecting from the shear layer of the supersonic plume; this is referred to as an annular separation bubble. In general the initial shocks are sufficiently strong such that an additional shock (emanating from the wall) will form which will separate the flow from the inner wall through a SWBLI mechanism. Presumably, the flow will reattach further downstream, thereby creating a second bounded annular separation bubble.
Subsequent bubbles can form this way. Upon further increases in NPR, the RSS structure translates downstream and the enclosed separation bubbles open up to ambient when passing over the nozzle lip. When the last annular separation bubble downstream of the incipient separation shock opens up, the flow returns to FSS state due to the presence of a single separation shock with an associated separated flow region downstream. The nozzle will eventually flow full -overexpansion shocks emanate from the nozzle lip- and achieves perfectly expanded flow at even higher NPR. The latter two do not influence the flow topography of the internal nozzle flow and are therefore of minor interest when studying start-up loads in high-area ratio nozzles.

The difficulty with these quasi-steady flow models however, is that they differ from the full-scale operating environment in the sense that the flow is transient, unsteady and three-dimensional (3-D) in nature. This increased level of complexity expresses itself in numerous features, for example (1) the azimuthal variation of separation lines, reattachment lines, shock structures, and annular separation bubbles, (2) unsteadiness $\mathrm{n}$ the shock waves and zones of separated flow, (3) linear and nonlinear interactions between flow structures that can possibly amplify or cancel globally unsteady loads, and (4) the intermittent opening to atmosphere of the last separation bubble that is driven by the reattachment line interacting spasmodically with the nozzle lip for a short period of time during start-up (roughly $0.5 \mathrm{~s}$ during the start of a full-scale engine). The latter one is referred to as the 'end-breathing effect' or 'end-effects regime' (Nave and Coffey, 1973; Nguyen et al, 2003). An example of the azimuthal dependence of these events is shown in Fig. 2, which was taken during the staggered start-up of several full-scale liquid propellant engines. The incipient separation line appears as a white line whose position varies significantly in azimuth.

Because these flows are transient, unsteady, and 3$\mathrm{D}$ in nature, a combination of techniques are implemented in this study under various operating conditions of a TOP nozzle and based on the wall pressure alone. The signatures registered at the wall provide insightful information regarding the location and dynamical characteristics of the shock foot and surrounding fluid, and so, they have been used in various studies throughout the literature to elucidate global features of this internal flow (Nguyen et al, 2003; Verma et al, 2006; Baars et al, 2012a). In this study, the spatially and temporally resolved wall pressure is first decomposed in azimuth ( $\S 4.1$ ) so that Fourier-azimuthal modes can be studied as opposed to isolated single-point measurements. This reduces the dimension of the system by allowing the azimuthal spatial structure to be repre- 

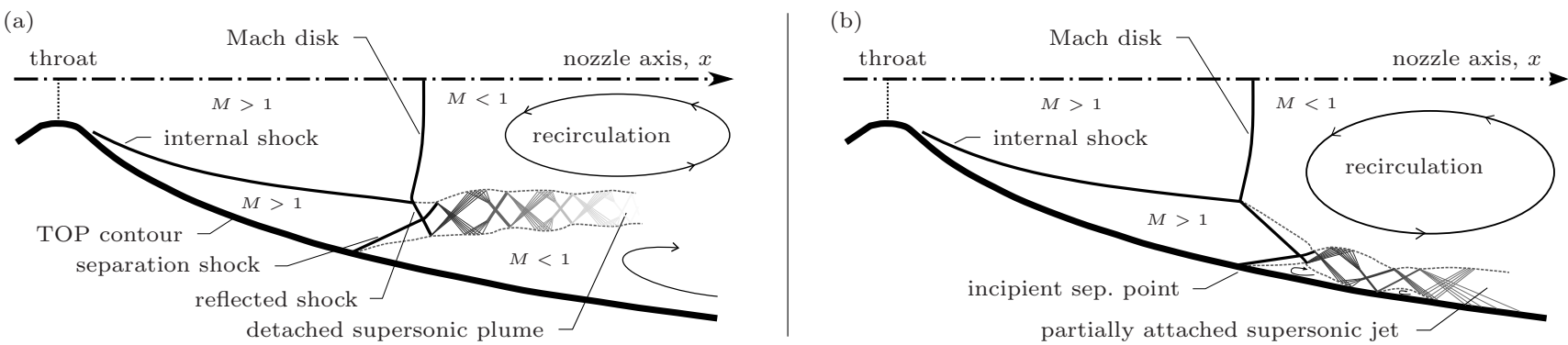

Fig. 1 Illustration of the internal shock structure in a TOP nozzle during a Free Shock Separation (FSS) state and b Restricted Shock Separation (RSS) state - following Baars et al (2012a)

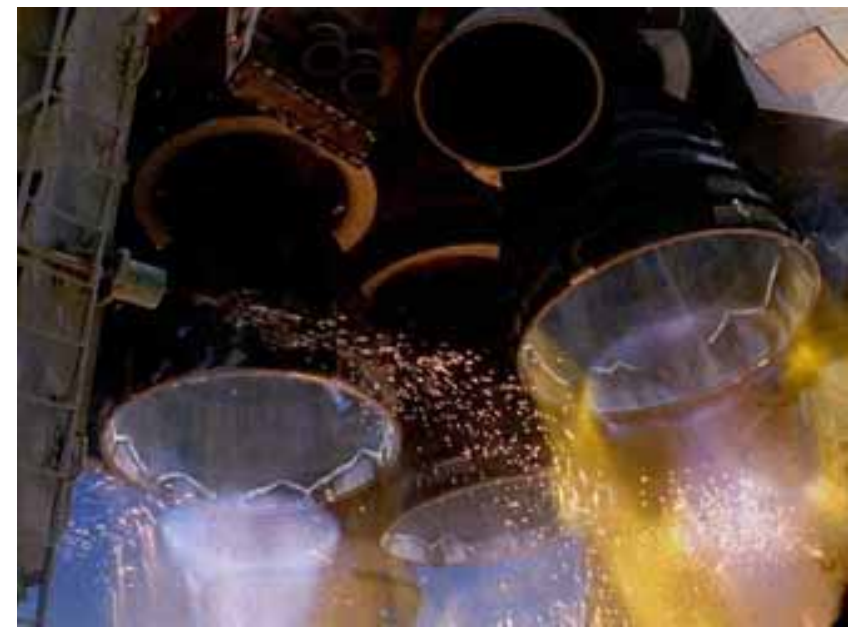

Fig. 2 Image of large-scale liquid propellent engines during start-up. Azimuthally dependent, incipient separation lines are depicted on the interior surfaces [courtesy: NASA].

sented by a discrete series of time-resolved mode coefficients. Secondly, the spectral features embedded in these time-resolved mode coefficients are then extracted to characterise the unsteady nature of the events. The approach is straightforward for stationary systems with spectra being generated through an ensemble-averaging process. In transient systems however, the mean signal is non-stationary and is much more challenging to handle as it requires that the temporal component be preserved. This is particularly difficult if the time scales associated with the important features in the signal reside on the same order of, or are larger than, the time scale of the transient episode. And so a persistent difficulty to developing statistical models of transient phenomena is simply the suitability of the analysis technique selected.

For highly-transient data, time-frequency analysis has the advantage of being able to characterize the spectral behavior of the signal as a function of time. This allows one to search for localized singularities, or regions in the time series, with unique spectral content. Such information is otherwise de-localized, i.e. spread out, when conventional harmonic analysis techniques are applied (Lewalle et al, 2000; Farge, 1992). Moreover, one could argue that the time-frequency analysis, as opposed to Fourier analyses involving ensemble averaging, is more realistic in the sense that engineering systems are rarely exposed to excitations described by ensemble-averaged spectra, but rather short bursts of energy. Joint time-frequency distributions and wavelet transforms ( $(4.2)$ provide mathematical frameworks for performing spectral decompositions in time and space and have been studied extensively by Cohen (1989) and Farge (1992), respectively.

Studies that focussed on the transient operation of rocket nozzles, such as the one by Verma and Haidn (2009), are limited by time-series analysis only. The first objective of this article is to compare the spectral footprints of the fluctuating wall pressure for fixed and transient NPRs to investigate whether an adequate understanding of the transient cycle (fluctuations superposed a transient mean flow) can be furnished by studying a sequence of steady flow analyses (fluctuations superposed a steady mean flow). Secondly, due to the available information of azimuthal mode energies in space and time, important conclusions are made concerning the end-breathing effect which has been known to cause the most energetic off-axis forces during the start-up of high-area ratio nozzles.

\section{Experimental arrangement}

\subsection{Facility and hardware}

The experimental campaign was conducted in the fully anechoic chamber and open jet facility of The University of Texas at Austin. All surfaces of the chamber are treated with 18 in melamine foam wedges followed by a 4 in air cavity and 5.5 in of recycled cotton fibre. This level of treatment provides a normal incidence sound absorption coefficient of $99 \%$ for frequencies above $100 \mathrm{~Hz}$. The dimensions of the chamber walls measure (wedge tip to wedge tip) $19 \mathrm{ft}(\mathrm{L}) \times 15 \mathrm{ft}(\mathrm{W})$ 


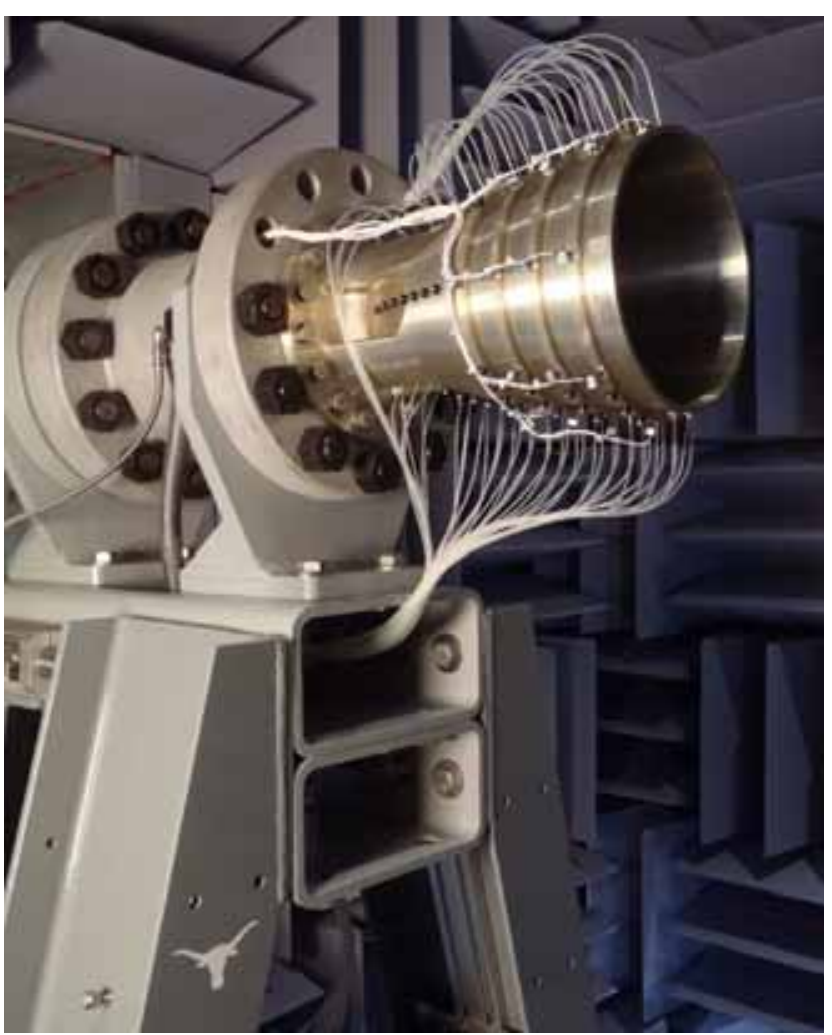

Fig. 3 The fully instrumented TOP nozzle installed in the fully anechoic chamber at The University of Texas at Austin

$\times 12 \mathrm{ft}(\mathrm{H})$. A custom fabricated nozzle test rig is installed along the centerline of the chamber, as shown in Fig. 3. Entrained air is allowed to enter the chamber through a $4 \mathrm{ft} \times 4 \mathrm{ft}$ opening behind the nozzle test rig which then exhausts through a $6 \mathrm{ft} \times 6 \mathrm{ft}$ acoustically treated duct followed by a $90^{\circ}$ elbow with turning vanes. A $500 \mathrm{hp}$ vane axial fan located at the aft end of the exhaust duct is operated at carefully selected speed settings required to prevent recirculations from forming inside the chamber.

Compressed air is stored at 2,100 psig by several tanks comprising $4.25 \mathrm{~m}^{3}$ of water volume storage that are charged between runs by an in-house compressor. These high pressure tanks are connected to the test rig through a 4 in pipe that expands to a 6 in diameter plenum located immediately upstream of the nozzle mounting flange. Nozzle pressure ratios $(N P R=$ $\left.p_{0} / p_{\infty}\right)$ are regulated using a 3 in pneumatically actuated control valve. The plenum houses a Corning Celcor $\AA$, ceramic flow straightener (400 square cells per $\mathrm{in}^{2}$ substrate) that conditions the flow before entering into the nozzle contraction. Nozzle operating conditions (plenum pressure $p_{0}$, ambient pressure $p_{\infty}$, ambient temperature $T_{\infty}$, plenum temperature $T_{0}$ ) are monitored and recorded using a NI CompactRIO system at a rate of $50 \mathrm{~Hz}$, which also serves as the control sys-
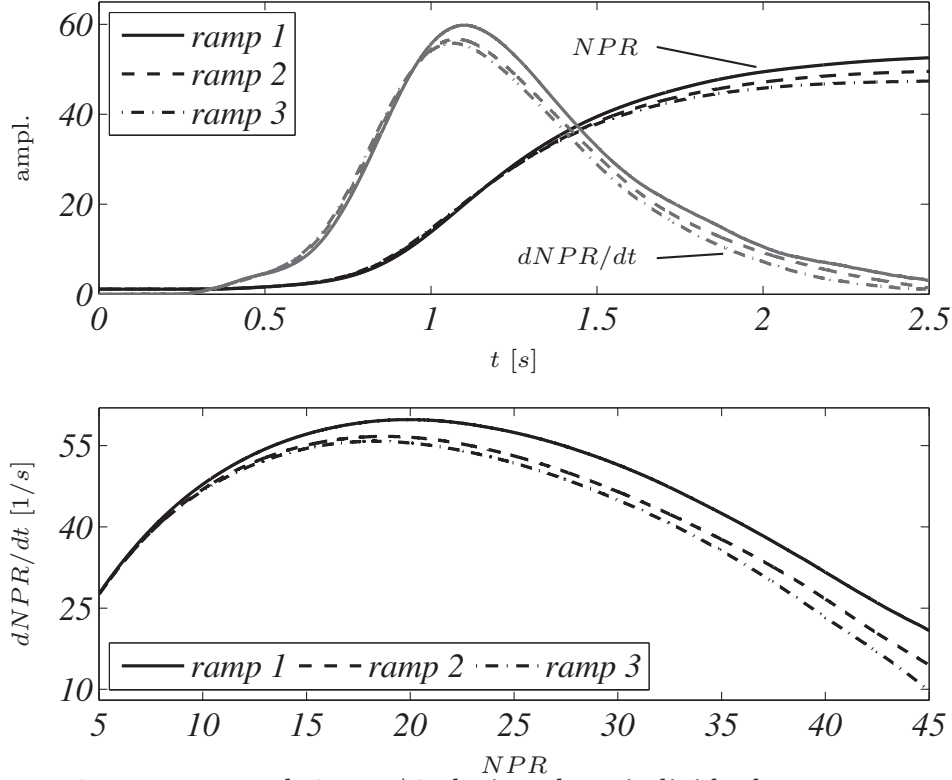

Fig. $4 N P R$ and $d N P R / d t$ during three individual start-up transients, denoted ramp 1-3

tem for the valve (open- or close-loop). Additional details concerning the facility are described by Donald et al (2012) and Baars et al (2012b). Cold-flow tests were performed using an axisymmetric TOP contoured nozzle on loan from The Nozzle Test Facility Team of NASA's Marshall Space Flight Center (Ruf et al, 2010). This nozzle test article was designed specifically to simulate the separated flow behavior observed during start-up of large liquid propellent rocket engines. The throat radius of the nozzle is $r^{*}=19.05 \mathrm{~mm}(0.75 \mathrm{in})$ and has an exit-to-throat area ratio of 38 (design exit Mach number of 5.58). The nozzle was manufactured from one solid piece of AL 6061-T6 with a maximum standard-of-deviation interior surface roughness of 35.2 $\mu$ in. The profile of the nozzles' interior contour is shown in Fig. 6 along with relevant coordinates.

\subsection{Experimental conditions}

The data reported here was separated into two categories: (1) transient NPR conditions (start-up only, $d N P R / d t>0)$ and (2) fixed NPR conditions. For transient conditions, three ramping rates were considered (ramp 1-3); their trends are shown in Fig. 4. Variations in these ramp rates are shown to occur whenever $N P R \gtrsim 10$.

Fixed nozzle operating conditions were chosen for NPR set points of 25, 30, 40 and 50 in order to replicate previous studies performed by (Ruf et al, 2010) and Baars et al (2012a). At each set point, data was acquired for a minimum of $5.1 \mathrm{~s}$ with variations in the 


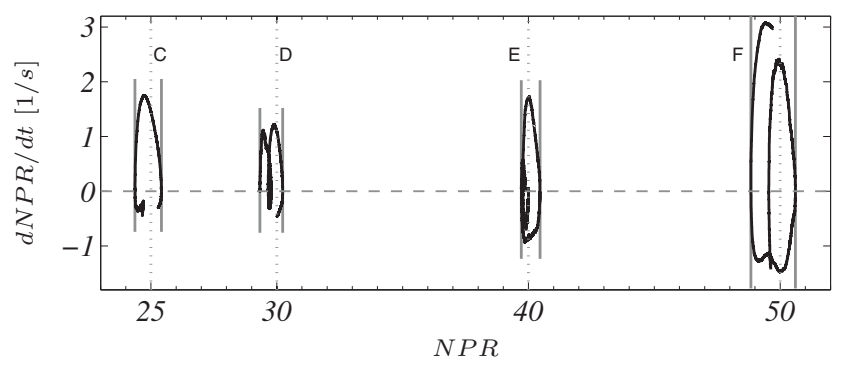

Fig. 5 Graph of $N P R$ and $d N P R / d t$ in time during the fixed NPR 25, 30, 40 \& 50 condition; the bounds of maximum deviation from the nominal NPR set points are indicated

nozzle operating conditions being illustrated by timetraces in Fig. 5.

Given the surface roughness of the test article, the boundary layer in the supersonic part of the nozzle is believed to transition naturally before interacting with the separation shock. The Reynolds number in the nozzle throat, based on throat diameter and isentropic compressible flow equations, depends linearly on NPR and is determined to be $R e_{d^{*}}=7.7 \cdot 10^{4} \cdot N P R$. Furthermore, the boundary layer is believed to have developed under near adiabatic wall temperature conditions.

\subsection{Instrumentation and acquisition}

The TOP nozzle test article was outfitted with both static and dynamic wall pressure ports. Static ports were oriented in the axial direction along a single curvilinear array at $\theta=90^{\circ}$, as shown by the white pressure tubing on top of the nozzle in Fig. 3. This array consisted of 32 static pressure ports ( $\varnothing 1.10 \mathrm{~mm}$ thru hole) with an equidistant axial spacing of $\Delta x / r^{*}=0.45$ spanning between $x / r *=3.85$ and 17.80 ; the exit plane of the nozzle is located at $x / r^{*}=18.40$. Static wall pressure profiles were acquired using two Scanivalve (R) DSA3218 Digital Pressure Scanners. Each module incorporates 16 temperature compensated piezoresistive pressure sensors that were digitized using 16 bit A/D converters. The absolute pressure sensors have a full scale (FS) range of 50 psia with an accuracy of $\pm .05 \% \mathrm{FS}$, and were calibrated down to 1.5 psia. Both Scanivalve systems were triggered synchronously at an acquisition rate of $440 \mathrm{~Hz}$ by an external TTL signal.

As for the arrangement of the dynamic wall pressure transducers, four axial stations, with various configurations in azimuth, were located at $x / r^{*}$ positions of $9.73,12.07,14.40$ and 16.73 (further referred to as $\left.x_{j}, j=1 \ldots 4\right)$. The first axial station had four ports $\left(\varnothing 2.64 \mathrm{~mm}\right.$ thru hole) in azimuth $\left(\theta=0^{\circ}, 90^{\circ}, 180^{\circ}\right.$ and $270^{\circ}$ ), whereas the three subsequent axial stations comprised eight ports in azimuth with equal increments
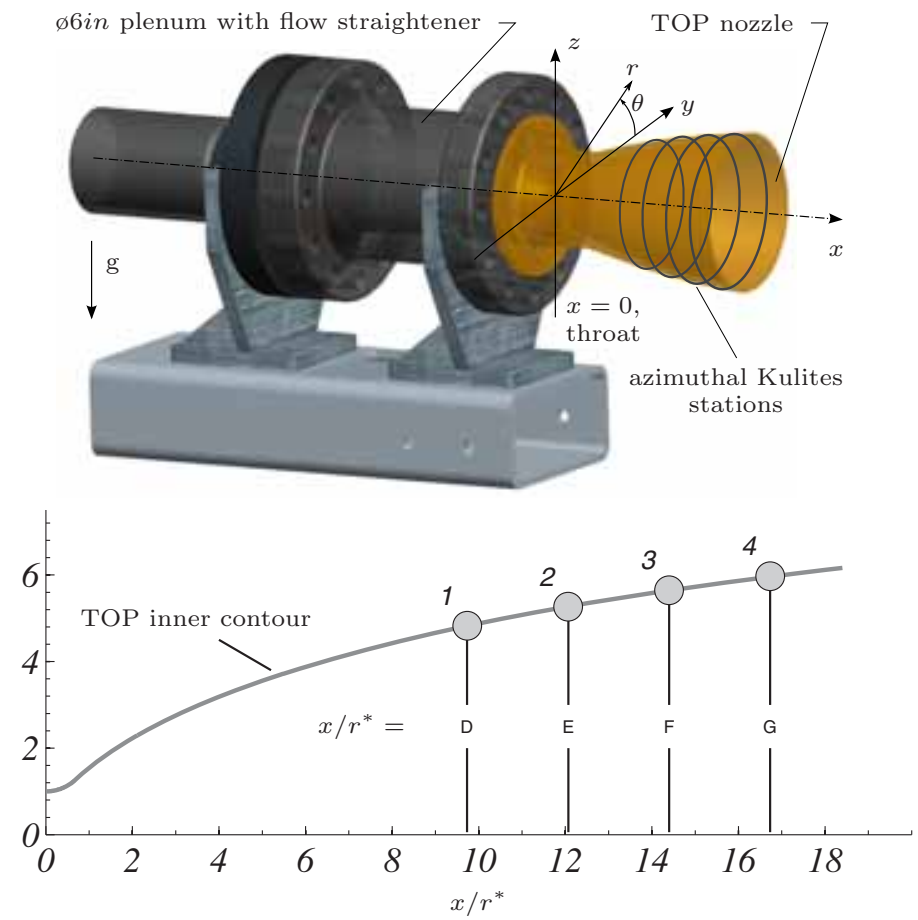

Fig. 6 (top) Schematic overview of the nozzle setup with coordinate system. (bottom) Locations of the azimuthal-oriented Kulites: station 1 includes four transducers $\left(\Delta \theta=90^{\circ}\right)$ and station 2-4 include eight transducers $\left(\Delta \theta=45^{\circ}\right)$

of $\Delta \theta=45^{\circ}$, starting at $\theta=0^{\circ}$ (28 dynamic wall pressure sensors in total). These dynamic transducers can be identified by their white colored wiring in Fig. 3; their positions relative to the nozzle contour are shown in Fig. 6. The transducers comprised high-frequency Kulite $\AA$ XT-140 models and were screwed directly into the nozzle wall (6-32 UNC thread), so that their protective screens ("B" screens with an outside diameter of $2.62 \mathrm{~mm}$ ) were mounted flush with the interior surface of the nozzle. These "B" screens allow for an effective frequency response range up to $20 \mathrm{kHz}$. The absolute transducer type of the Kulite XT-140 family was selected with a dynamic pressure range of 100 psia, infinitesimal resolution and a manufacturer specified accuracy of $\pm 0.1 \%$ FSO. Four National Instruments ${ }^{R}$ NI PXIe-4331 bridge input modules (embedded in a NI PXIe-1073 chassis) were used to provide the necessary power, filtering and signal conditioning for operating the 28 transducers through four NI TB-4330 terminal blocks. All channels were sampled simultaneously at a rate of $40 \mathrm{kHz}$, with 24 bit A/D conversion per channel. All three acquisition systems (static pressure, dynamic pressure, nozzle operating conditions) were synchronized using the same $440 \mathrm{~Hz}$ TTL signal that was used to trigger the Scanivalve systems. 


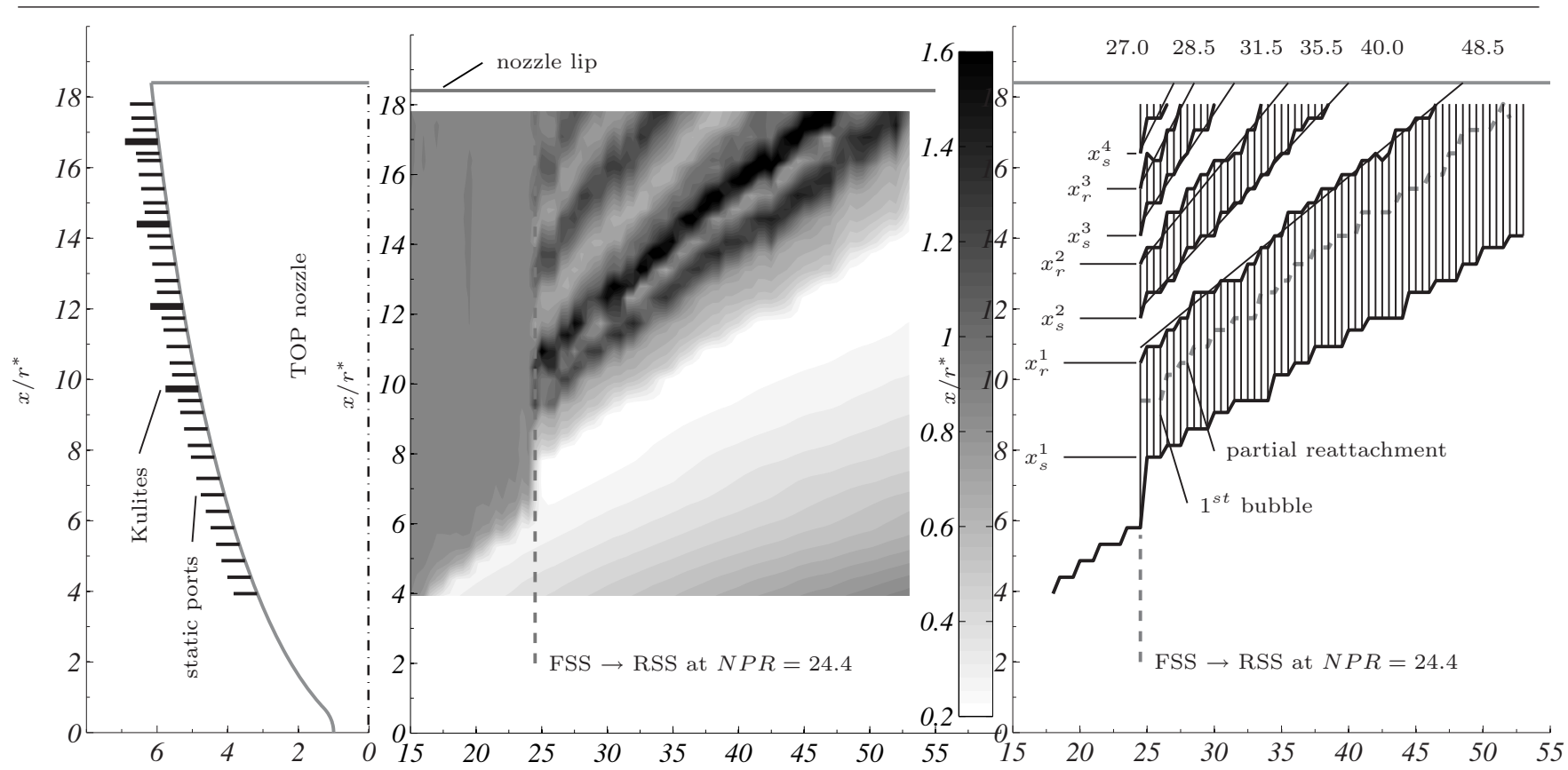

Fig. 7 a Schentatic of the TOP nozzle contour and the Rocations of the static and dynamic pressure ports, $\mathbf{b}$ Contours of the static wall pressure $p / p_{\infty}$ as function of NPR during a slow start $\left(d N P R / d t \approx 5 \mathrm{~s}^{-1}\right)$, c Illustration of the separation and reattachment lines based on figure $\mathrm{b}$ and the approximate NPRs at which these reach the nozzle lip

\section{Internal flow topography}

Static wall pressure profiles of the TOP nozzle are shown in Fig. 7 under quasi-steady operating conditions. The ramping rate of the nozzle is slow enough (approximately $5 \mathrm{NPR} / \mathrm{s}$ ) so that the flow can be considered steady, all the while allowing for changes in the static wall pressure to be visualized. This test was separate from the fixed and transient conditions described earlier and was conducted solely for the purposes of constructing Fig. 7. A visual interpretation of the RSS flow structure is also provided in Fig. 8 during low NPR conditions in order to assist with the discussion of these results.

The position of the static and dynamic wall pressure ports are shown in Fig. 7a and alongside the static wall pressure footprint in Fig. 7b. For each NPR, the static wall pressure profile was constructed by windowaveraging over an interval spanning 0.5 NPR. The pressure footprint illustrates well known features corresponding to various operating states of a TOP nozzle. For example, at low NPR (below 24.4), the flow is in a FSS state which is characterized by isentropic expansion of the flow up to the incipient separation line. Hereafter the pressure rises to near ambient levels due to the formation of a subsonic entrainment region. Transition is found to occur here at NPR $\sim 24.4$ at which the incipient separation line translates aft due to the less resistive nature of the downstream flow (i.e. an attached supersonic jet versus low speed entrained reverse flow). Pressure maximas and minimas reveal a number of separation lines (denoted by $x_{s}$ ), reattachment lines $\left(x_{r}\right)$, and separated flow regions, following the illustration in Fig. 8; the reader is referred to Baars et al (2012a) for a detailed interpretation of these pressure profiles. In Fig. 7c, the pressure profiles are reconstructed to illuminate regions of attached (white) and separated flow (hatched). Separation bubbles are denoted by $1^{\text {st }}, 2^{\text {nd }}$ and $3^{\text {rd }}$, with the $1^{\text {st }}$ bubble located furthest upstream. Labels on the top of the figure identify the NPR value at which the $x_{s}$ and $x_{r}$ lines reach the nozzle-lip (extrapolated linearly), e.g. the $1^{\text {st }}$ bubble opens up to atmosphere at a NPR of about 48.5. A 'partial reattachment' line is drawn and corresponds to a local pressure maxima in the $1^{\text {st }}$ bubble. This is believed to be associated with the impingement of expansion fans on the nozzle wall. It is important to recognize that the wall pressure profile distribution is dependent on ramp rate. When increasing the ramp rate considerably from this quasi-steady analysis, delay- and hysteresis-effects are expected to occur which can influence the activity at the wall (Nguyen et al, 2003).

Ensemble-averaged wall pressures from the azimuthal arrays of Kulites during the four fixed NPR conditions are presented in Fig. 9. Averaged values from all axial stations are shown to coincide with the static wall pressure signatures shown in Fig. 7. Because the Kulites are absolute type transducers, their outputs are expected to 

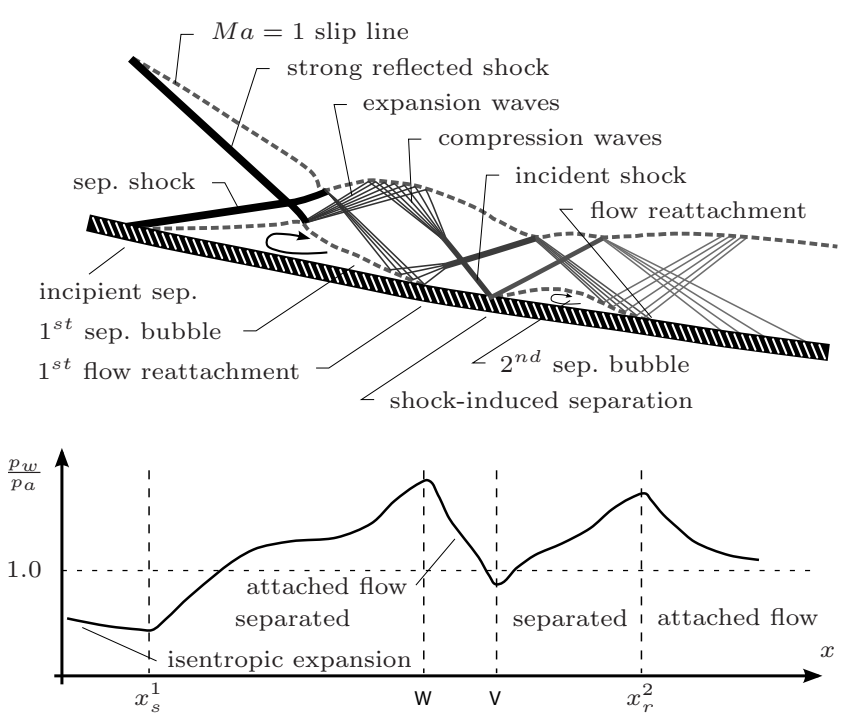

Fig. 8 Detailed overview of the RSS internal shock. A typical time-averaged wall pressure profile is indicated below extracted from Baars et al (2012a)

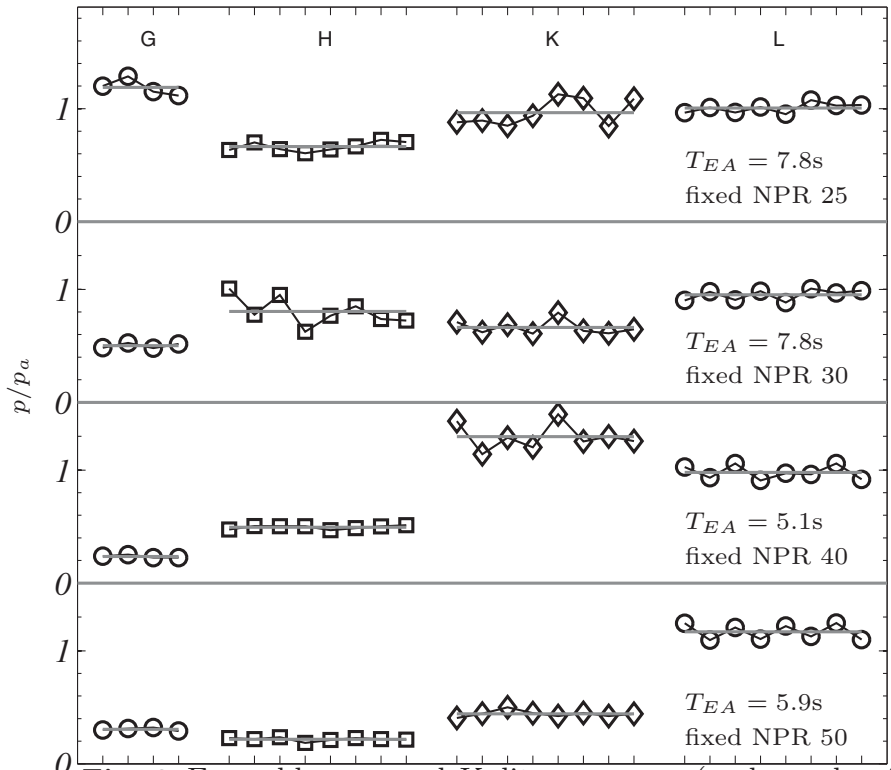

0 Fig. 9 Ensemble-averaged Kulite pressures (each marker is one transducer at that specific station). The time of averaging $T_{E A}$ is listed on the right. The grey line indicates the mean wall pressure at each station.

match that of the static wall pressure system. During different NPR set points of the nozzle, signatures corresponding to the location of the separation and reattachment lines reside at different positions along the nozzle wall as shown in Fig. 7. Closer inspection reveals how an average of the fluctuating wall pressure footprint manifests a stationary dependence in azimuth when the reattachment side of the first separation bubble $x_{r}^{1}$ resides in close proximity to axial station $x_{2}$ at NPR $30, x_{3}$ at NPR 40, and $x_{4}$ at NPR 50. The averaging process was scrutinized for convergence and repeated for smaller time samples. For stations located further upstream in the $1^{\text {st }}$ bubble and near the separation line, the average static pressure reflects less stationary dependence in its azimuthal variation. This does not necessarily mean that the separation line is non responsive to finer variations in azimuth. For example, strong tipilike shock patterns observed in Fig. 2 during the startup of a large-scale liquid propellent engine revealed a non-stationary azimuthal dependence in their location when viewed at high frame rates. This provides some explanation for the near axisymmetry in the static wall pressure near the separation shock for $x_{1}$ at NPR 30, $x_{2}$ at NPR 40, and $x_{3}$ at NPR 50. Similar features were found in the transient data. Likewise, the full-scale image in Fig. 2 illustrates fine azimuthal variations in the shock pattern, which cannot be resolved with the current 8-point grid. And so, it is likely that the azimuthal shock pattern is much finer than what is being resolved here. It is postulated that the triple-point shock interaction and resulting outward expansion fan (that causes the flow to reattach to the nozzle wall) is more receptive to azimuthally unstable events in the flow, which in turn, causes the reattaching shock wave to vary significantly in azimuth. The upstream separation shock however is exposed to a relatively undisturbed incoming flow. It is worth pointing out that the azimuthal variation of $x_{r}^{1}$ does not necessarily furnish an off-axis load to the nozzle, as the resultant azimuthal pressure distribution may still be axisymmetric. In fact, it will be shown in $\S 4.1$ how most of the dynamic azimuthal energy resides in the axisymmetric mode. As for the NPR 25 data, certain caution should be exercised in view of the lack of convergence in these measurements. Albeit, this suggests a slow variation (on the order of $1-10 \mathrm{~Hz}$ ) in the subsonic entrainment region which is believed to be the consequence of a low-frequency azimuthal mode residing in the detached supersonic jet.

\section{Analyses of the dynamic pressure}

\subsection{Fourier-azimuthal decomposition}

Insightful information about the unsteady three dimensionality of the flow and shock structure interactions can be gleaned by Fourier transforming the original high-frequency pressure field, $p(x, \theta, t)$, in azimuth to obtain time-dependent Fourier-azimuthal mode coefficients

$p\left(x ; m^{\prime} ; t\right) \in \mathbb{C}, \quad p\left(x ; m^{\prime} ; t\right)=\mathcal{F}[p(x, \theta, t)]_{\theta}$,

where $m^{\prime}$ indicates the mode number. These spatial coefficients have units of psia. $\sqrt{\mathrm{rad}}$. Recall that for both positive $\left(\mathrm{m}^{\prime}=1,2,3\right)$ and negative $\left(\mathrm{m}^{\prime}=-1,-2,-3\right)$ mode 
numbers, the complex Fourier coefficients form conjugate pairs, i.e. $p\left(x ; m^{\prime}=1 ; t\right)=p^{*}\left(x ; m^{\prime}=-1 ; t\right)$, while for $m^{\prime}=0 \& 4$, these coefficients are real. In the remainder of this work, the real $(m>0)$ and imaginary $(m<1)$ parts of the Fourier coefficients $m^{\prime}=0,1,2,3,4$ are considered and are denoted as

$p(x ; m ; t) \in \mathbb{R}, \quad m=0,1,-1,2,-2,3,-3,4$.

These 'mode coefficients' represent the amplitude of the physical mode shapes illustrated in Fig. 10. The significance of having done this transformation is to allow multi-point variations in azimuth to be described by a reduced set of time dependent coefficients. Since the first Fourier-azimuthal mode, $m=1$ and $m=-1$, is the only one responsible for generating off-axis loads in the $x$ - and $y$-directions, the technique provides an opportunity to filter out other, potentially less threatening, mode number events from the full signal.

The total resolved energy embedded in all modes and at each axial station $x_{j}$, is computed as a function of time $k$ as follows

$\Lambda_{k}\left(x_{j}\right)=\sum_{m} \lambda_{k}^{(m)}\left(x_{j}\right)$,

where $\lambda_{k}^{(m)}\left(x_{j}\right)$ is the variance of the time-dependent mode coefficient $p\left(x_{j} ; m ; t\right)$ and $t \in k$. Fractions of resolved energy per mode are determined using

$\alpha_{k}\left(x_{j} ; m\right)=\frac{\lambda_{k}^{(m)}\left(x_{j}\right)}{\Lambda_{k}\left(x_{j}\right)}$.

The temporal domain $k$ used to compute $\alpha_{k}\left(x_{j} ; m\right)$, and under fixed operating conditions of the nozzle, is taken over the entire acquisition length. Slow drifts in NPR during fixed operating conditions were filtered out from the wall pressure data beforehand in order to correctly assess the energy in each azimuthal mode. Normalized Fourier-azimuthal mode eigenspectra are shown in Fig. 11a and 11b for axial stations $x_{3}$ and $x_{4}$, respectively. Likewise, the total resolved Fourierazimuthal energy in the fluctuating wall pressure for each operating condition is shown in Fig. 11c. Here we show how the fraction of energy in the breathing mode $(m=0)$ at station $x_{4}$ increases by a factor of almost three between the NPR 40 and 50 operating states of the nozzle. This is associated with the end-breathing effect which will be discussed in $\S 6.2$. The reader is referred to $\S 5$ for a more detailed discussion of Fig. 11.

\subsection{Time-frequency analysis}

One of the most appropriate tools for characterizing the spectral content embedded within a transient signal is known as joint time-frequency analyses, a thorough review of which can be found in the literature (a)

(b)
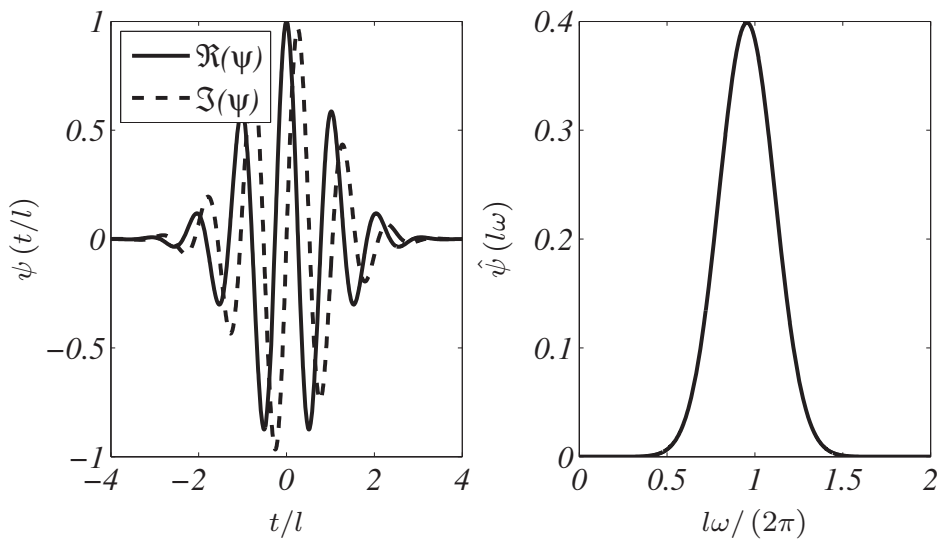

Fig. 12 The Morlet wavelet in a the time domain, $\psi \in \mathbb{C}$, and $\mathbf{b}$ the frequency domain

(Cohen, 1989; Farge, 1992; Addison, 2002). The continuous wavelet transform is applied in the current work to the real-valued pressure mode coefficients $p(x ; m ; t)$ to apprise its space-time-frequency behaviour.

\subsubsection{Morlet wavelet transform}

The wavelet transform of a signal is performed by convolving a known function (mother wavelet) with the signal. This convolution is performed at various time scales $l$, i.e. frequencies, and so decomposes the signal in time-frequency space. The progressive complex-valued Morlet wavelet, Eq. (5), is selected for this study due to its high frequency resolution when compared to other conventional wavelets. This helps identifying the energy in relatively narrow frequency bands, as was observed in the recent study of Baars et al (2012a). However, in choosing the Morlet wavelet, less temporal resolution is achieved when compared to, for example, the Mexican hat wavelet; this is particularly noticeable at the lowfrequency (large-scale) end of the spectrum. By correctly interpreting the time-frequency results (Addison, 2002) and referring back to the raw time signal, this obstacle can be overcome. A concise comparison between the obtained results for our application via both the Morlet and Mexican hat wavelet is discussed in $\S 6$ and presented in Fig. 17.

$\psi(t / l)=e^{j \omega_{\psi} t / l} e^{-|t / l|^{2} / 2}$

The Morlet wavelet comprises a simple plane wave whose amplitude is modulated by a Gaussian function. The non-dimensional frequency is taken as $\left|\omega_{\psi}\right|=6$. Both the Morlet wavelet and its transform are shown in Fig. 12. The temporal convolution of the wavelet with each of the fluctuating wall pressure Fourier-azimuthal mode coefficients produces a set of complex-valued wavelet 

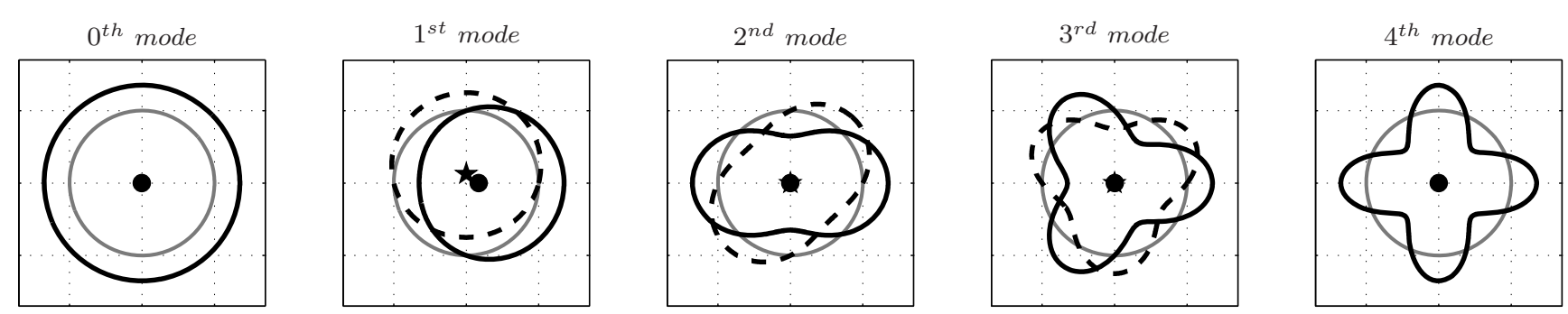

Fig. 10 Physical interpretation of the Fourier-azimuthal decomposition. Solid and dashed lines correspond to the real $(m>0)$ and imaginary $(m<0)$ parts of the Fourier coefficients

(a)

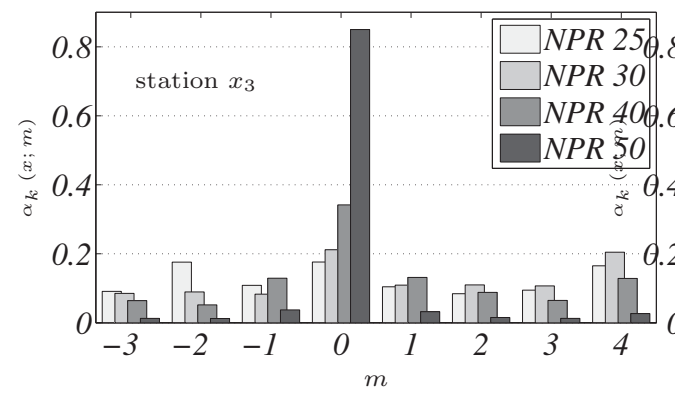

(b)

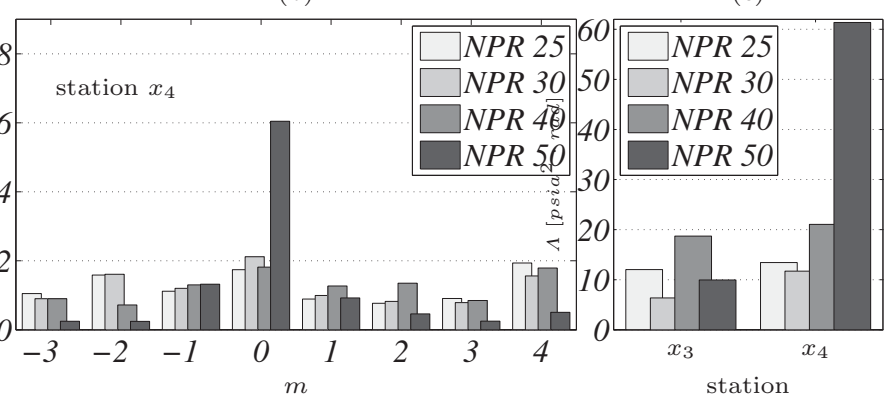

Fig. 11 Scaled eigenspectra of the Fourier-azimuthal modes at station $\mathbf{a} x_{3}$ and $\mathbf{b} x_{4}$. c Total resolved Fourier-azimuthal energy in the fluctuating wall pressure at each operating condition of the nozzle

coefficients $\tilde{p}(x ; m ; l, t)$ defined as,

$\tilde{p}(x ; m ; l, t)=\int p\left(x ; m ; t^{\prime}\right) \psi^{*}\left(\frac{t^{\prime}-t}{l}\right) d t$,

with the convolution being performed in the frequency domain (Farge, 1992). Wavelets are mutually similar amongst the scales to ensure similar influence on the coefficients. This results in relatively sparse temporal resolution at large scales (low frequencies) but with fine temporal resolution in the smaller scales (high frequencies). In the current study, the resolved band of frequencies range between $10 \mathrm{~Hz}<f<f_{s} / 2$ using a base- 2 logarithmic progression of 81 scales in order to achieve a uniform grid on a logarithmic scale. The energy density is given by

$E(x ; m ; l, t)=\frac{|\tilde{p}(x ; m ; l, t)|^{2}}{l}$,

and is known as the Wavelet Power Spectrum (WPS). As a last step, the wavelet scale is transformed to an equivalent Fourier frequency, i.e. $E(x ; m ; l, t) \rightarrow E(x ; m ; f$ The WPS of a sample Fourier-azimuthal mode coefficient $p\left(x_{3} ; m=1 ; t\right)$ during a fixed NPR of 40 is shown in Fig. $13 \mathrm{a}^{1}$.

Trigonometric basis functions are used to create Fourier spectra, which are therefore de-localized (this prevents

1 Overlapping signal partitions of $N=2^{14}$ samples are transformed. The regions inside the cone of influence (Farge, 1992), for $10 \mathrm{~Hz}<f<f_{s} / 2$, are presented in a continuous fashion throughout the article. singularities in the data from being preserved), as opposed to the localized wavelet analysis (Farge, 1992). A Fourier based singe-sided power spectral density, computed for the same signal, is presented in non-dimensional form as $G(x ; m ; f) \cdot f / \sigma^{2}$ in figure $13 \mathrm{~b}$ where $\sigma^{2}$ is the signal's variance. For wavelet analyses, when time scales of transient events are small, time-averaged wavelet spectra can be created following $\bar{E}=1 / T \int_{T} E d t$, where $T$ is an arbitrary domain in time. If the signal is steady, the single-sided global wavelet spectrum $(G W S=2 \cdot \bar{E}$, and $\mathrm{T}=$ length of signal) compares well with the ensemble averaged Fourier spectra, as is shown in Fig. 13b.

\subsubsection{Sensitivity to the transient ramp rate}

An illustration of how the nozzle ramp rate affects the pressure footprint along the interior surface of the nozzle is presented in Fig. 14 for the $m=0$ azimuthal $f$, thode. Each column corresponds to a unique ramp rate (top figure) which decreases slightly for each subsequent ramp from left to right. The temporal signal corresponding to this breathing mode $(m=0)$ is shown at the bottom of figure 14. In order to compare the results from three different ramp rates, a linear NPR scale is chosen for the $\mathrm{x}$-axis. Wavelet power spectra are then shown in the middle illustration with a logarithmic scale for the amplitude being used to accommodate for the large spread in energy that occurs over the ramp range 

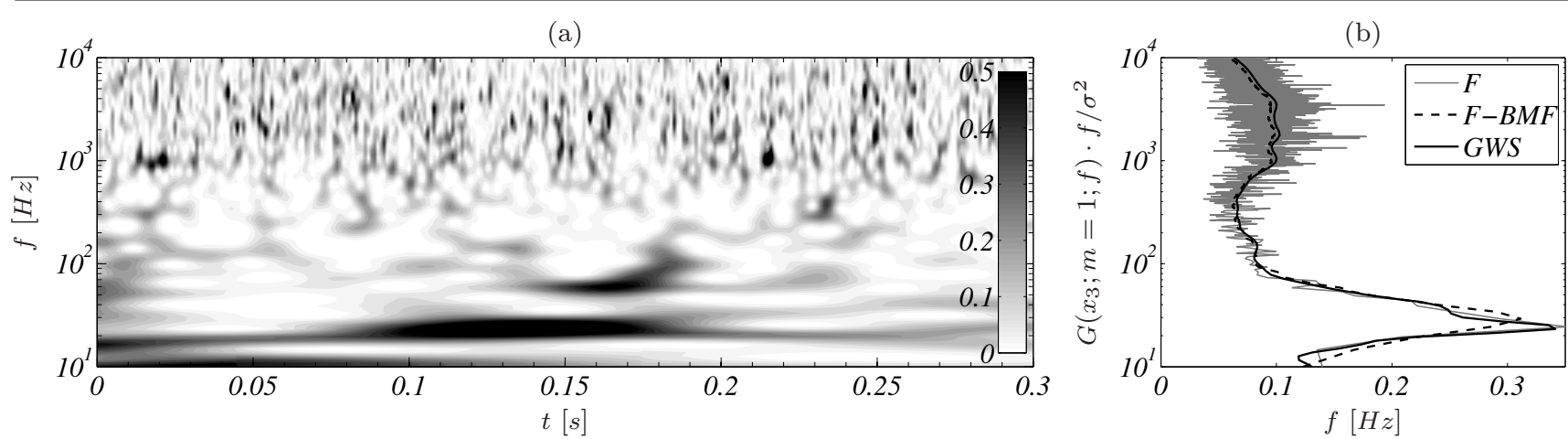

Fig. 13 a Wavelet power spectrum $2 \cdot E\left(x_{3} ; m=1 ; f, t\right) \cdot f / \sigma^{2}$ of the fixed NPR 40 condition, b Comparison of the global wavelet spectrum (GWS) and the Fourier spectrum (F) (both computed from $4.8 \mathrm{~s}$ of data) - a $5 \%$ bandwidth moving filter is

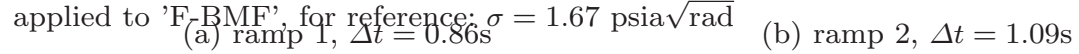
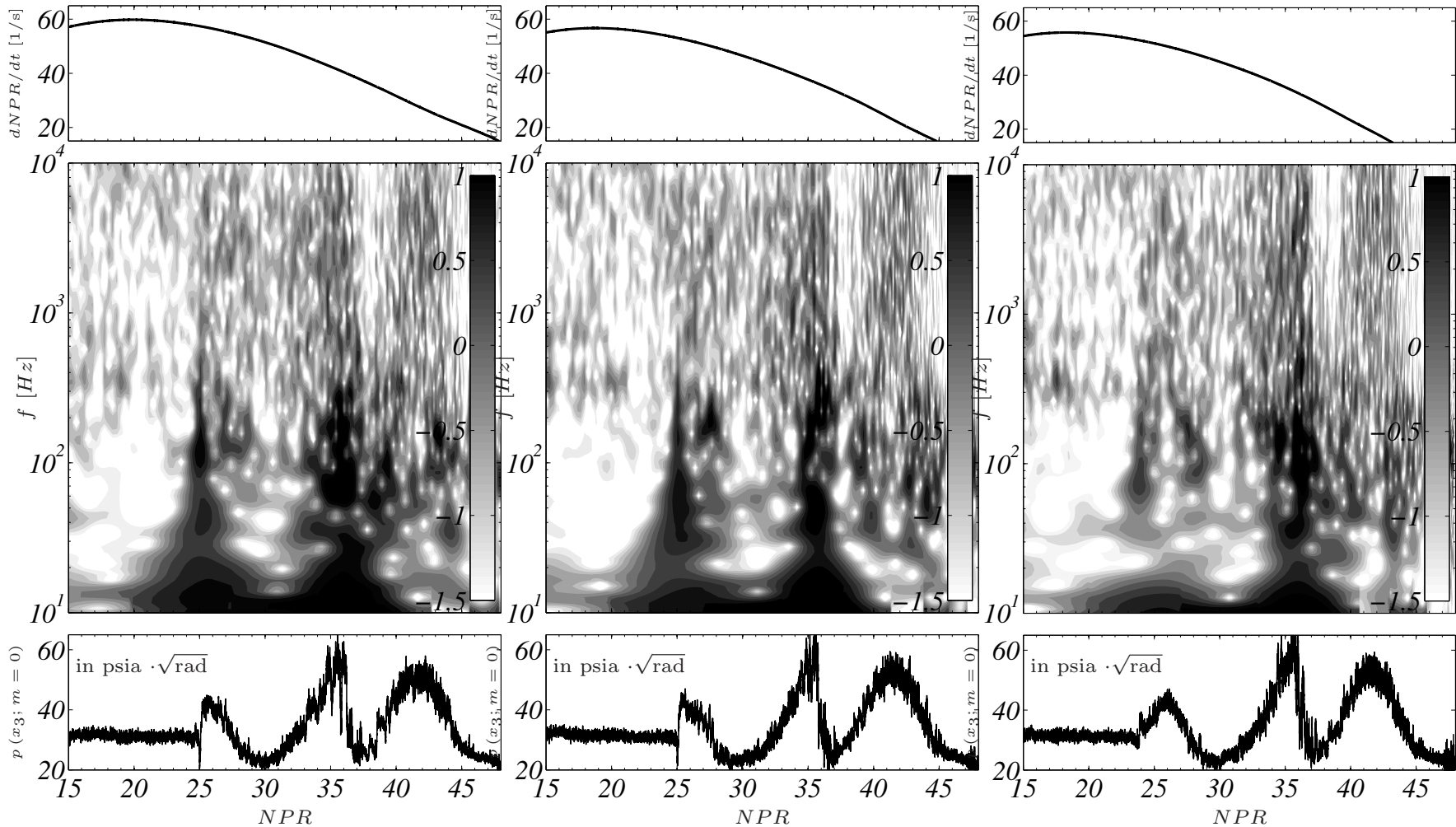

Fig. 14 Wavelet power spectra, plotted as $\log _{10}\left(E\left(x_{3} ; m=0 ; f, t\right) \cdot f\left[\mathrm{psia}^{2} \mathrm{rad}\right]\right)$ as function of NPR. The ramp rate (top) and raw time signal (bottom) are shown with the wavelet transform (middle) of the raw time signal. $\Delta t$ is the total duration of time encompassing the NPR range shown

being investigated (NPR 15 to 48). FSS $\rightarrow$ RSS transition is revealed by an abrupt spike in the raw time signal during the first two ramps, just prior to NPR 25, while the transition results in a more smooth signal during the slower, third ramp. Upon transitioning to an RSS state, separated and attached flow regions alternately pass over this station $\left(x_{3}\right)$ which produce peaks and valleys in the temporal breathing mode pressure signature. Where the wavelet power spectra are concerned, two high intensity events are observed. The first being FSS $\rightarrow$ RSS transition, which is relatively void of signif- icant spectral energy in the $15 \mathrm{~Hz}$ to $50 \mathrm{~Hz}$ band during ramp 3. The second most intense broadband frequency event occurs around NPR 35. Again, the slower ramp rate is necessarily devoid of the same spectral energies that are observed in the faster two ramp rates. Following the illustration in Fig. 7c, it is concluded that this second high energy event is affiliated with the passing of the reattachment line of the $1^{\text {st }}$ separation bubble. It is postulated that this increased unsteadiness near the reattachment location is the result of a greater abundance of turbulent/shock-wave unsteadiness upstream 
of the expansion waves that cause the flow to reattach (thus trapping the bubble), as well as multiple shock/expansion-wave/slip-line interactions adjacent to the reattachment. The separation shock encompasses much less dynamic motion due to the lower levels of upstream flow unsteadiness.

From an engineering point of view, it would be useful to know the influence that the transient ramp rate has on the total resolved energy that the system is exposed to. This is done by integrating the global wavelet spectrum (essentially the energy integrated over time) over all resolved frequencies, according to

$E_{W}(x ; m)=2 \int_{10}^{f_{\max }}\left\{\frac{1}{T} \int^{T} E(x ; m ; f, t)\right\} d f$,

where $f_{\max }$ is confined to $10 \mathrm{kHz}$. Following Parseval's theorem, this would be the variance of a stationary signal, and so, Eq. (8) can be interpreted as the variance of a transient signal. A factor of two has been included since the wavelet spectrum is double-sided. Upon determining $E_{W}\left(x_{3} ; m=0\right)$ for the three ramps, ramp 2 was found to be $\approx 16 \%$ less energetic than ramp 1 , and ramp 3 was $\approx 44 \%$ less energetic than ramp 1 (see table 1). This is in line with earlier observations by Baars et al (2011) of the same nozzle conducted under relatively constant ramp rates ranging between $8 \mathrm{NPR} / \mathrm{s}$ and $25 \mathrm{NPR} / \mathrm{s}$. It should be pointed out that the measurements used in the analysis of Baars et al (2011) were acquired in a vacuum chamber where the FSS $\rightarrow$ RSS transition was observed to occur at a much higher NPR ( 30) than what is found in the current study (24.4). And so, it is difficult to make a direct comparison between the current study and the findings from Baars et al (2011).

If one now considers the total energy that the system is exposed to (essentially $E_{W} \cdot \Delta t$, where $\Delta t$ is the duration of the ramp indicated in Fig. 14), it is apparent that this value is largest for ramp 3 and lowest for ramp 1. This is simply the result of the duration of these ramps. A general observation from this is that only subtle deviations in the ramp rate are required to have a considerable influence on the amount of energy that the system is exposed to, while the energy in the fluctuations increases with increasing ramp rate. Since the above observations are only valid for one mode coefficient $(m=0)$ and axial location $\left(x_{3}\right)$, the next logical step is to determine these trends for all other coefficients and locations along the nozzle wall. The total wavelet energies for the first three mode coefficients at locations $x_{3}$ and $x_{4}$ are summarized in table 1 . It is clear that the observations of mode $m=0$ at $x_{3}$ do not extend to other mode numbers. The mode number energy is shown here to both increase and decrease with decreasing ramp rate. Baars et al (2011) found that
Table 1 Total wavelet energies $\left(E_{W}\right.$, in psia $\left.^{2} \mathrm{rad}\right)$ in each mode coefficient during start-up. The difference in percentage with respect to ramp 1 is shown in parentheses.

\begin{tabular}{rrrcc}
\hline$x$ & mode & ramp 1 & ramp 2 & ramp 3 \\
\hline \multirow{4}{*}{$x_{3}$} & 0 & 11.36 & $9.50(-16 \%)$ & $6.40(-44 \%)$ \\
& 1 & 1.72 & $1.90(+11 \%)$ & $1.12(-34 \%)$ \\
& 2 & 2.08 & $1.53(-26 \%)$ & $1.23(-41 \%)$ \\
\hline \multirow{4}{*}{$x_{4}$} & 0 & 10.03 & $14.41(+44 \%)$ & $33.34(+233 \%)$ \\
& 1 & 3.16 & $2.31(-27 \%)$ & $3.13(-1 \%)$ \\
& 2 & 2.62 & $2.88(+10 \%)$ & $2.86(+9 \%)$ \\
\hline
\end{tabular}

higher fluctuations occurred for slower ramp rates. This trend was only tested for a smaller domain encompassing FSS $\rightarrow$ RSS transition. Therefore, while the spectral behavior is generally similar between runs, the overall energy acting on the system is less predictable during transient operations of the nozzle.

\section{Transient versus fixed operating conditions}

In order to compare the dynamical characteristics between transient and fixed NPR conditions, the total resolved energy of the mode coefficients are inspected. For fixed nozzle operating conditions, the fraction of energy in each Fourier-azimuthal mode was presented in Fig. 11. As for transient conditions, where the nozzle is operated under the three ramp rates illustrated in Fig. 4, the temporal behavior of the $m=1$ mode coefficient is shown in Fig. 15a for all three ramps. The fraction of energy in each mode (Eq. 4) is then computed for NPR conditions corresponding to 25, 30 and 40 by averaging over a window with a width of 1 NPR that is centered around the three targeted NPRs. These temporal mode coefficients were high-pass filtered at $10 \mathrm{~Hz}$ before computing $\alpha_{k}(x ; m)$. Eigenspectra corresponding to the first two Fourier-azimuthal modes $(m=0$ and $m=1$ ) are shown in Fig. 15 for the $x_{3}$ and $x_{4}$ axial stations and for all three ramps (denoted 1-3) alongside the eigenspectra obtained from fixed (denoted fix) NPR conditions. Here we see that for all cases studied, the $m=0$ breathing mode dominates the energy wavenumber spectra of the fluctuating wall pressure and is in line with the observations of Baars et al (2011). However, a large discrepancy in the $m=0$ mode energy is shown to occur at NPR 25 where the first two ramps are shown to comprise a greater portion of energy than the third ramp and fixed NPR condition. This is attributed to the FSS $\rightarrow$ RSS transition which has been shown to occur around NPR 24.4 in this study. And so, it is believed that transition occurred toward the tail end of the averaging window for ramp 3 (more FSS type flow), as opposed to ramp 1 and 2 (more RSS type flow). The 
(a)

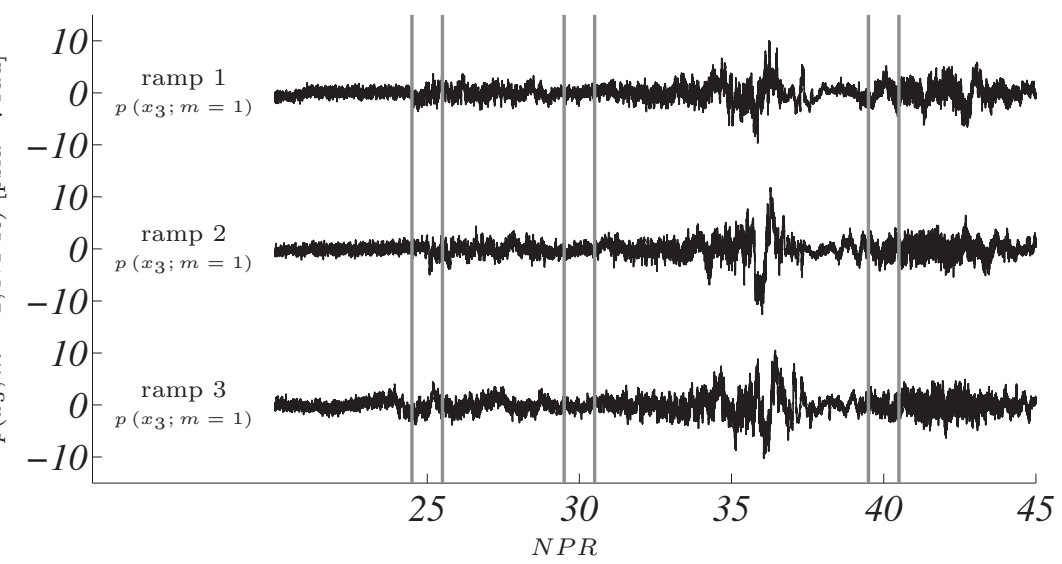

(b)

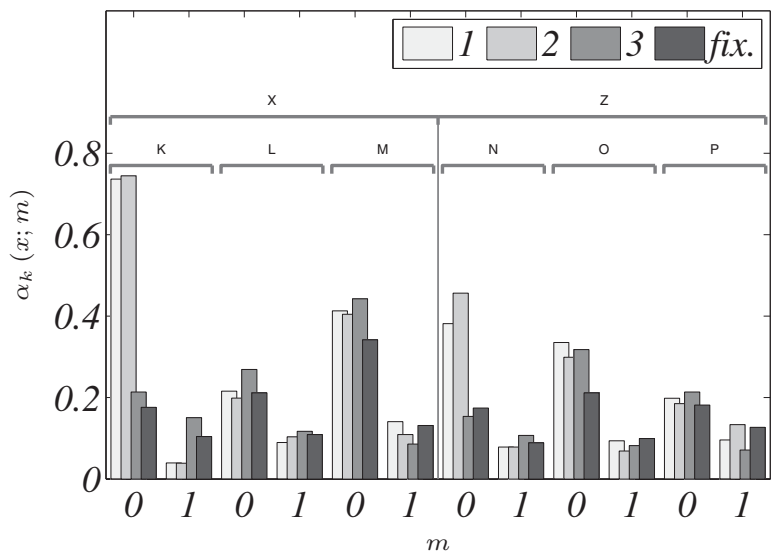

Fig. 15 a Fourier-azimuthal mode coefficient $(m=1)$ at axial station $x_{3}$ in $[\mathrm{psia} \cdot \sqrt{\mathrm{rad}}]$ as function of NPR for three different ramps. b Scaled eigenspectra of the $m=0$ and $m=1$ Fourier-azimuthal modes for three different ramps (1-3) alongside fixed NPR conditions (fix.)

smaller fraction of energy in the $m=0$ mode during the FSS $\rightarrow$ RSS transition in ramp 3 is in agreement with earlier observations in Fig. 14c.

Having now compared the energy in the Fourierazimuthal modes, it is of interest to understand whether the spectral behavior of these pressure modes, observed during fixed NPR operations, reflect the same dynamical characteristics observed during transient (start-up) operations of the nozzle. This was the underlying objective of the study by Baars et al (2011), where the same sub-scale TOP nozzle was tested in a vacuum type flow facility with an ejector diffuser that was found to cause $\mathrm{FSS} \rightarrow \mathrm{RSS}$ transition to occur at NPR $\sim 30$. In that environment, the steady state operating conditions $(25,30,40$ and 50) comprised both flow states, e.g. FSS only during NPR 25 and RSS only during NPR 40 and 50. Here, our testing of the same nozzle in an open jet facility at NPR 25 comprises an RSS flow structure, and so the current study is limited by the lack of steady-state data under FSS conditions of the flow. Baars et al (2011) generated several 'instantaneous' spectra at NPR 25 and 40 by time-averaging the wavelet power spectra over windows centred on the NPR set points. At NPR 25, the ramp rate appeared to have little influence on the fluctuating wall pressure structure; albeit, the transducers were located in the subsonic entrainment regions of the FSS flow. Under RSS conditions at NPR 40, the spectral distribution was highly sensitive to $d \mathrm{NPR} / d t$. Furthermore, it was shown that the window width had a direct influence on the instantaneous spectra and demonstrated how even modest amounts of temporal averaging had a profound influence on the resultant spectra. A better understanding of this filtering would comprise a relationship between the time scale of the ramp and the time scales associated with the characteristic frequencies in the flow (Nguyen et al, 2003). An ensemble average over a number of identical transients, though impractical, would eventually converge to produce a unique spectral shape, thus allowing the effects of $d \mathrm{NPR} / d t$ to be more clearly understood. Nevertheless, it is clear from the analysis of Baars et al (2011) that FSS flow states reflect the same spectral characteristics during both steady and transient operations of the nozzle. However, the same conclusions could not be drawn for NPR's associated with the RSS regime, and so, it was suggested that a satisfactory understanding of the dynamical characteristics of the internal flow could not be furnished by way of a fixed flow (NPR) approach.

\section{Evolution of the separated flow}

6.1 Space-time frequency behavior of the wall pressure

Here we focus on understanding the time-frequency behavior of the fluctuating wall pressure at two axial locations in the flow, namely, $x_{3}$ and $x_{4}$. Since these measurements were synchronized, the spatial and temporal evolution of the wall pressure spectra can be analyzed by way of time-frequency analysis during start-up of the nozzle. In Fig. 16, the time-series of the breathing mode coefficient and its corresponding wavelet power spectra are shown for locations $x_{3}$ and $x_{4}$. Note that the color scale in Fig. 16a and 16b are equal. Various features associated with the near-wall flow topography have been included to assist with the discussion and are based on the static wall pressure footprint illustrated in Fig. 7c. For example, the $2^{\text {nd }}$ separation bubble passes over location $x_{4}$ when the NPR rises from 32.2 to 35.8 . 


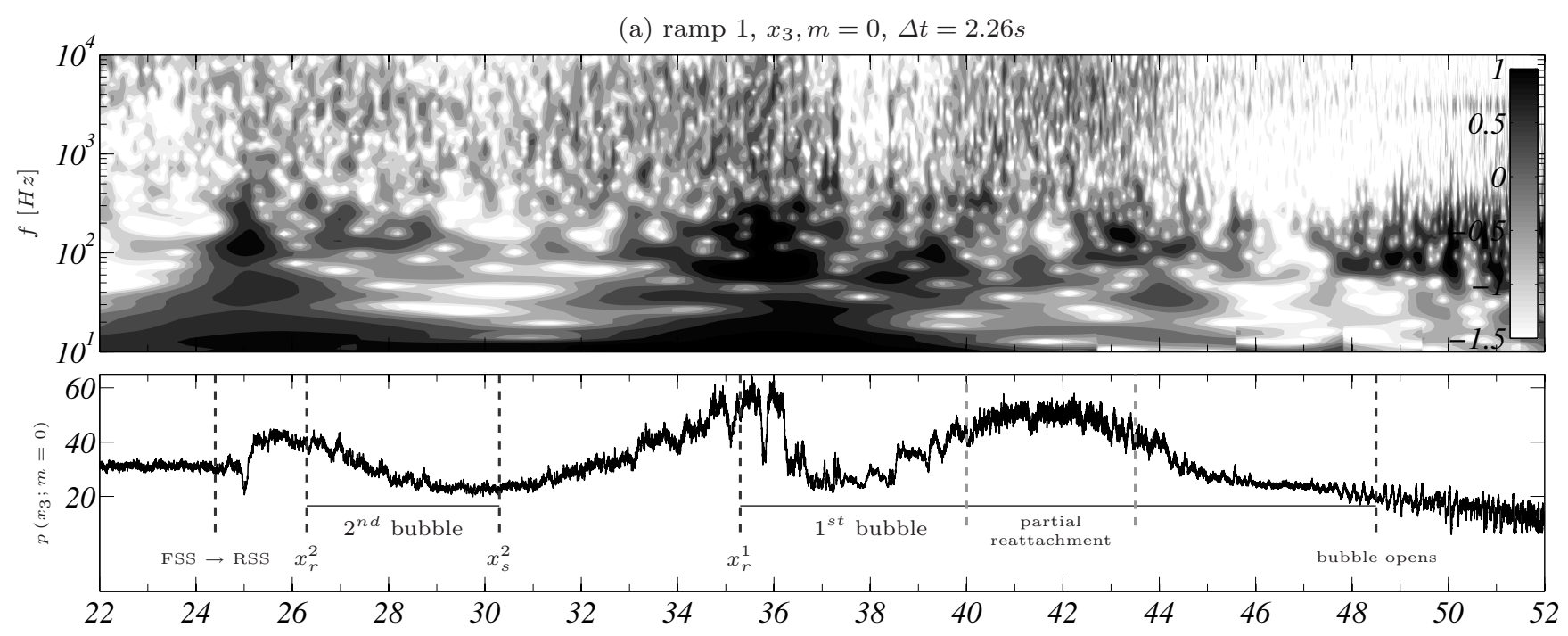

(b) $\operatorname{ramp} 1, x_{4}, \stackrel{P}{P}=0, \Delta t=2.26 s$

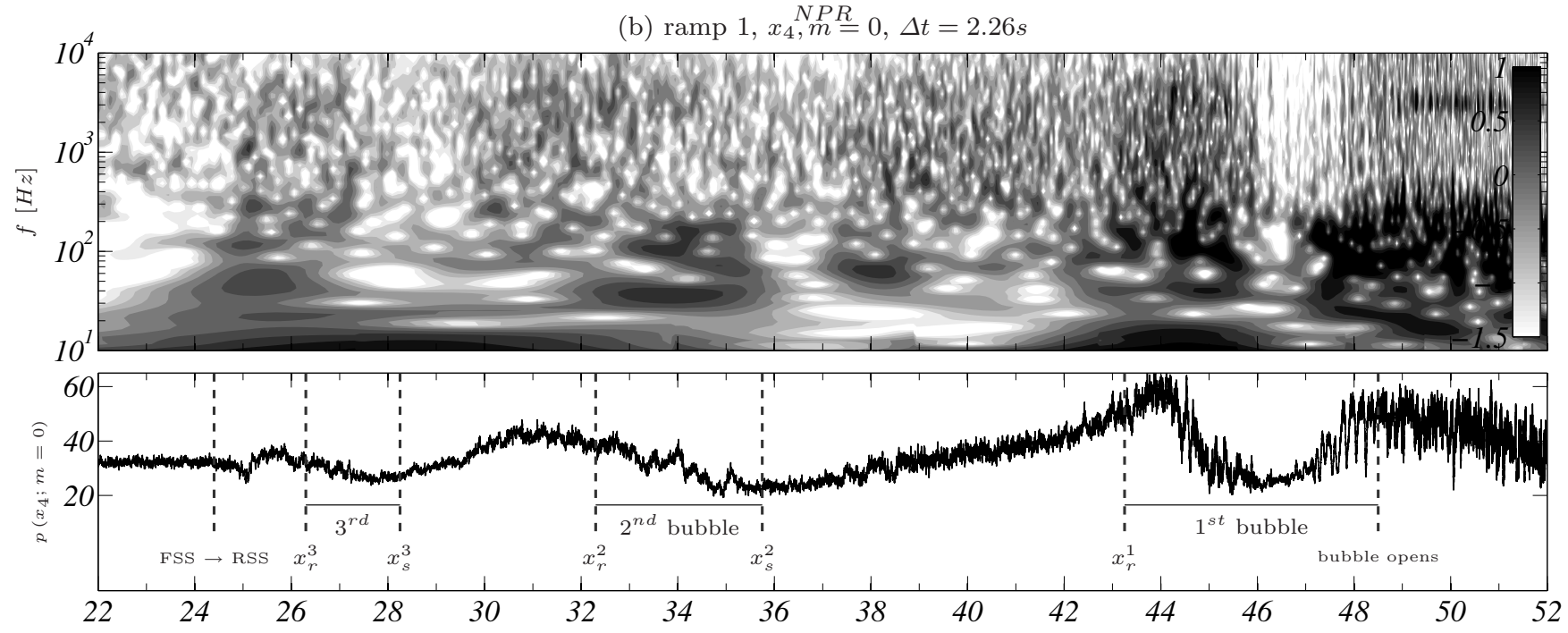

Fig. 16 Morlet wavelet power spectra plotted as $\log _{10}\left(E(x ; m \stackrel{N}{=} \stackrel{P}{0} ; f, t) \cdot f\left[\mathrm{psia}^{2} \mathrm{rad}\right]\right)$ at a $x_{3}$ and $\mathbf{b} x_{4}$. The raw time signal is synchronously shown. $\Delta t$ is associated with the NPR range

In Fig. 14, we showed how the first most energetic event occurs when the flow undergoes FSS $\rightarrow$ RSS transition. This event is more intense at the location closest to the transition point, namely $x_{3}$ where the transducer array is closest to the shock foot. With subsequent increases in NPR, the $2^{\text {nd }}$ and $3^{\text {rd }}$ separation bubbles pass over locations $x_{3}$ and $x_{4}$, respectively. Relative to the passage of the $1^{\text {st }}$ separation bubble (starting at NPR 35.5 at $x_{3}$ and then NPR 43.3 at $x_{4}$ ) the separation and reattachment lines of the $2^{\text {nd }}$ and $3^{\text {rd }}$ separation bubbles are low in energy and appear to decrease in their spectral intensity as they move further downstream. As for the most intense $m=0$ mode behavior, this is found to occur with the passing of the $1^{\text {st }}$ bubble's reattachment to the wall which is shown to manifest a band of frequencies centered around $100 \mathrm{~Hz}$. When this $1^{\text {st }}$ bubble eventually opens to ambient, a third high in- tensity event is observed with a more narrow band of frequencies residing around $150 \mathrm{~Hz}$. This is the so-called 'end-effects regime' which will be discussed in more detail in $\S 6.2$.

As for the space-time frequency behavior of the side load inducing mode ( $m=1$ azimuthal mode), its topography is shown in Fig. 17 from the same data set used to construct Fig. 16. Here the $\mathrm{FSS} \rightarrow \mathrm{RSS}$ transition and the passage of the $2^{\text {nd }}$ and $3^{\text {rd }}$ separation bubbles generate negligible activity. However, significant increases in side-load activity are observed with the passage of the reattachment line corresponding to the $1^{\text {st }}$ bubble $\left(x_{r}^{1}\right)$, as well as the end-effects regime at $N P R>48.5$. A typical comparison between the Morlet and Mexican hat wavelet power spectra are shown in Fig. 17b and Fig. 17c, respectively. As expected (see $\S 4.2)$, the Morlet wavelet smears the lower frequency 

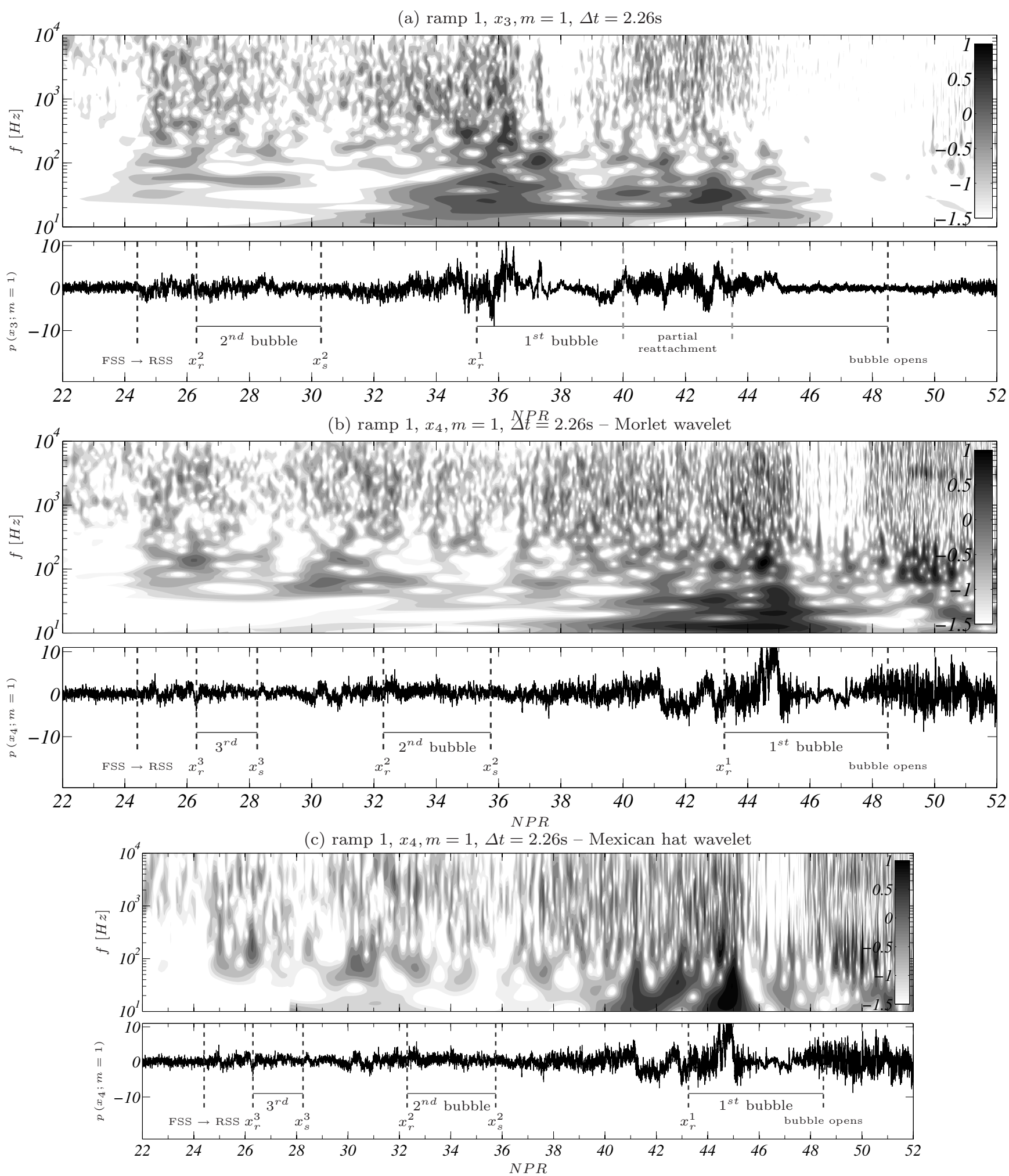

Fig. 17 Morlet wavelet power spectra plotted as $\log _{10}\left(E(x ; m=1 ; f, t) \cdot f\left[\mathrm{psia}^{2} \mathrm{rad}\right]\right)$ at $\mathbf{a} x_{3}$ and $\mathbf{b} x_{4}$. The raw time signal is synchronously shown. $\Delta t$ is the duration associated with the NPR range. c Mexican hat wavelet power spectrum for the same signal as in (b) 


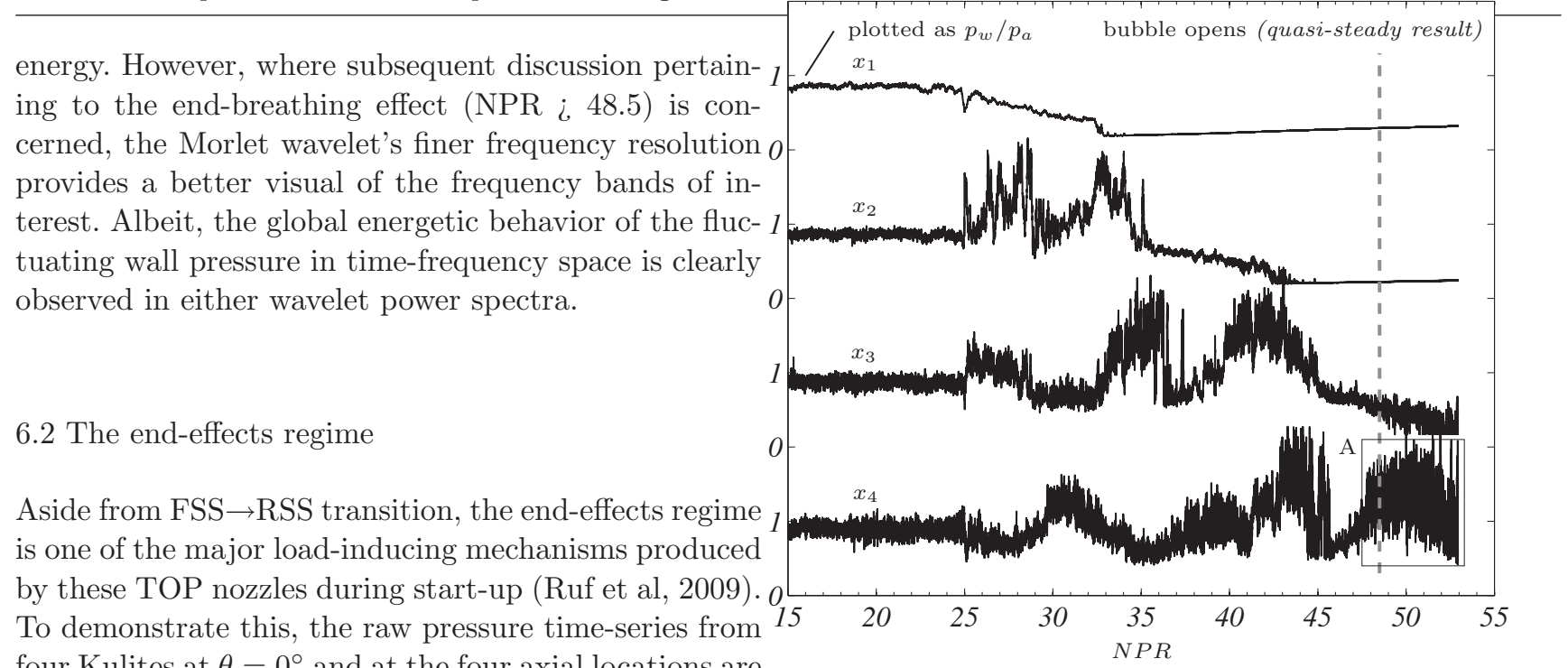

four Kulites at $\theta=0^{\circ}$ and at the four axial locations are shown in Fig. 18. The peaks and valleys at station $x_{2}$ and $x_{3}$ correspond to the RSS flow structure translating downstream and over the transducers as the start-up progresses; this is similar to the time-series presented in Fig. 14, except we are no longer viewing the fluctuating wall pressure in terms of their Fourier-azimuthal modes. The $x_{s}^{1}$ shock separation line moves over the $x_{1}$ and $x_{2}$ transducers at approximately NPR 33 and 44, respectively. A dashed line has been inserted at NPR 48.5 to identify the instant in time when the $1^{\text {st }}$ bubble opens to ambient, (based on the quasi-steady analysis in Fig. 7c), beyond which the fluctuating wall pressure at $x_{4}$ experiences massive unsteadiness (window A). This indicates that the end-effects regime is present (Nave and Coffey, 1973; Nguyen et al, 2003). This mechanism has been described as the intermittent opening of the initial separation bubble with atmospheric pressure, and is driven by spasmodic interactions of the reattachment line (incoming expansion waves) with the nozzle lip.

Considering now the modal energies that characterize this end-effects regime, we begin by reviewing Fig. 11a where $35 \%$ of the resolved energy in the $1^{\text {st }}$ trapped (before opening to ambient) separation bubble at location $x_{3}$ and fixed NPR 40 resides in the breathing mode $(m=0)$. As this trapped separation bubble eventually makes its way downstream and opens up to atmosphere (now at a fixed NPR of 50), the energy in the breathing mode increases significantly to $83 \%$ and $62 \%$ at location $x_{3}$ and $x_{4}$, respectively. Likewise, the total resolved energy almost triples. These findings are based on fixed operating states of the nozzle. Where transient operation of the nozzle is concerned, (ramp 1, which reaches NPR 50), the breathing mode was found to comprise $88 \%\left(x_{3}\right)$ and $61 \%\left(x_{4}\right)$ of the total resolved energy with the bubble opened to the atmosphere, as opposed to $35 \%\left(x_{3}\right)$ when the bubble was trapped

Fig. 18 Raw Kulite pressure signals at $\theta=0^{\circ}$ for ramp 1; the end-effects regime is indicated by window $\mathrm{A}$

(NPR 40, Fig. 11a). This demonstrates how this endbreathing regime is highly unsteady, but is relatively symmetric. However, as the separation line upstream of the opened separation bubble comprises a stationary variation in azimuth (see $\S 3$ ), the absolute energy contained in the side-load inducing mode $(m=1)$ is highest during NPR 50, since the absolute energy (in $p i^{2} \mathrm{rad}$ ) is the product of the mode fraction energy with the total resolved energy. It is this high absolute energy that is responsible for causing the $0.25 \mathrm{~s}-0.5 \mathrm{~s}$ of violent shaking and excitation of the nozzle wall deflection modes observed during the start-up of high area ratio nozzles [personal communication with Joseph H. Ruf].

The next step to understanding this end-effects regime is to consider the spectral characteristics of the Fourierazimuthal pressure modes near the nozzle lip. Fig. 19a and $19 \mathrm{c}$ present single-sided PSD's of the eight individual Kulites at location $x_{4}$ during NPR 40 (attached flow) and NPR $50\left(1^{\text {st }}\right.$ opened separation bubble, i.e. end-effects regime). PSD's of the first three Fourierazimuthal mode coefficients (frequency-dependent eigenspectra) are also computed for the same 8 transducers and are shown in Fig. 19b and 19d. From Fig. 19b it can be seen that when the flow is attached, the broadband distribution of energy is confined mostly by a breathing mode structure, but with non-negligible contributions from the $m=1$ and $m=2$ modes and over the same range of frequencies. On the other hand, when considering conditions where the end-effects regime is present (NPR 50), it is clear that the fluctuating wall pressure is completely saturated by the axisymmetric breath- 


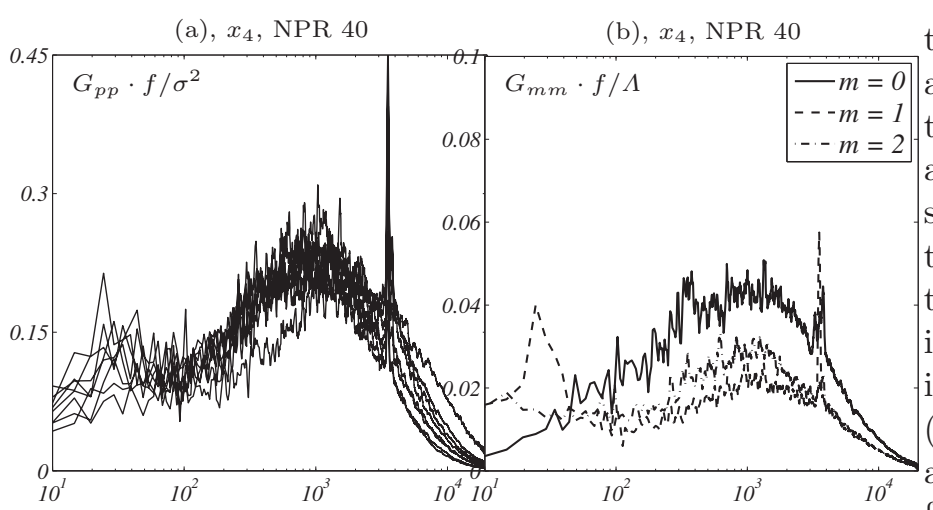

tion of highly overexpanded nozzles. While these tones are most often encountered in nozzles with area discontinuities, they can be found in smooth walled nozzles and nozzles without abrupt area changes. Shock unsteadiness directly influences transonic resonance, since the shock motion acts as a vibrating piston which drives the downstream flow (Zaman et al, 2002). The concept is comparable to classical problems in acoustics pertaining to standing waves that form in an open ended pipe (Blackstock, 2000). For a conical duct that is driven by a source located at the smaller of the two diameters, the fundamental acoustic resonance forms from one-quarter

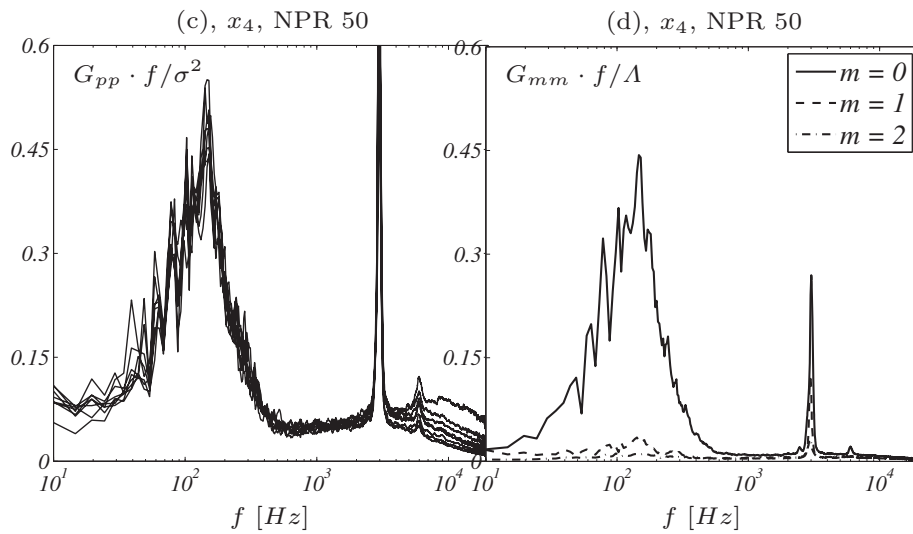

Fig. 19 Single-sided power spectral densities from all 8 individual pressure transducers at station $x_{4}$ during a NPR 40 and c NPR 50. b,d Frequency-dependent eigenspectra of the first three Fourier-azimuthal modes at $x_{4}$ during a NPR 40 and $\mathbf{c}$ NPR 50

ing mode. Here we see that the peak frequency resides around $160 \mathrm{~Hz}$ and was also observed in the wavelet spectra contours in Fig. 16b at NPR's larger than 48.5 during transient operations of the nozzle.

The formation of narrow spectral peaks in the PSD's of Fig. 19 was unexpected, given that these measurements were conducted in an acoustically treated facility. The data used in the analysis of Baars et al (2012a) comprised similar tones but was acquired in a vacuum chamber with hard walls located in close proximity to the nozzle. These tones were believed to be the consequence of facility induced interactions with the nozzle. However, their presence in the current data set suggests that they are attributed to a feature of the nozzle and/or operating condition; we now attribute these tones to either screech or transonic resonance. Without having directly measured the flow, it is not possible to infer a primary screech frequency from the shock cell structures in the plume. And so, we will consider only the case of transonic resonance as described by Zaman et al (2002).

Transonic resonance is the emission of acoustic tones by flow resonance that forms within the divergent sec-

of the standing wave pattern, or any of its odd harmonics. A first-order approximation of this standing wave's frequency is determined by dividing the sound speed of the traveling wave by the wavelength of the tone. During NPR 50, this tone occurs when the separation bubble has become pressure balanced with the ambient, and so the sound speed of the gas is that of ambient conditions $\left(a_{0}=344 \mathrm{~m} / \mathrm{s}\right)$. As for the wavelength of the tone $(L)$, this is measured as the distance between the incipient separation shock (node) and the nozzle lip (anti-node) and by assuming that a $1 / 4$-wavelength (or one of its odd harmonics) resides in that region. Therefore, from Fig. $7 \mathrm{c}$, we find that $L \approx 0.09 \mathrm{~m}$ for NPR 50, which results in a $1 / 4$-wave resonance tone of $a_{0} /(4 \cdot L)=956 \mathrm{~Hz}$. The spectral peaks in Fig. 19c coincide quite well with the first odd harmonic of this resonance tone $3 \cdot 956=2,868 \mathrm{~Hz}$, thus suggesting that transonic resonance at this NPR is plausible. The situation is more complicated at NPR 40 since the RSS flow structure, with a trapped separation bubble, is attached to the wall; a characteristic length is then difficult to define.

\section{Concluding Remarks}

A sub-scale, axisymmetric, high-area ratio TOP nozzle was investigated experimentally to gain a better understanding of the unsteady wall pressure during transient operation. Both static and dynamic wall pressure data were acquired for four fixed NPRs $(25,30,40 \& 50)$ and were compared to three start-ups where the NPR was ramped up to $\sim 50$. Under these conditions, the nozzle operates in a highly overexpanded state that is governed mostly by RSS flow. Spatially and temporally resolved wall pressure data were first decomposed in azimuth so that Fourier-azimuthal modes could be studied as opposed to isolated single-point measurements. An analysis of the time-frequency behavior of the various mode coefficients was then performed using the continuous Morlet wavelet transform. 
From a sequence of four fixed NPR conditions, the pressure signature registered within close proximity to the reattachment line of the first bubble comprised stationary variations in azimuth, as opposed to, for example, the incipient separation shock that is believed to be highly non-stationary. Images of full-scale engines undergoing start-up suggest that this separation shock is characterized by non-stationary, high mode number activity. This shock motion is attributed to a sensitivity of the shock triple-point and expansion fans to downstream flow unsteadiness (dynamic distortion). Albeit for these stationary azimuthal variations, it was shown by way of Fourier-azimuthal decomposition that most of the energy resides in the axisymmetric breathing mode $(m=0)$. This was consistent for all fixed NPRs investigated, as well as transient start-ups.

As for the transient study, it was shown how slight deviations in ramp rate can considerably influence the amount of energy that the nozzle wall is exposed to, even though the general spectral and temporal features remain similar. During start-up, three major low-frequency $(f \lesssim 400 \mathrm{~Hz})$ events were observed: (1) FSS $\rightarrow$ RSS transition, which is most energetic near the incipient separation point in the downstream region, (2) the passage of the reattachment line of the first bubble, and (3) the 'end-effects regime'. The last one refers to the phase at which the first separation bubble opens to ambient, in an intermittent sense, and was characterized by low frequency unsteadiness around $160 \mathrm{~Hz}$. The above observations affirm that the low-frequency phenomena induce extreme off-axis loads on the nozzle wall and support structure, despite the fact that the phases are relatively axisymmetric.

Acknowledgements The authors are grateful to Joseph H. Ruf and the NTF team members at NASA-MSFC for their assistance in this project. Funding for this study was provided graciously by AFOSR under award number FA955000-0-0203 (Dr. John Schmisseur as Technical Monitor) and from the Space Shuttle Main Engine Project, NASA Engineering and Safety Center, the Space Shuttle Loads Panel (Edward Burns as Technical Monitor). Full-scale imagery was provided by Robert Scharf, Chris Cloudt \& Marco Lozano of ESCG in support of the Image Science and Analysis Group of NASA-JSC. Special thanks to Brian Donald, Alexis Avram and Lauren Cooper for their assistance during the experimental campaign.

\section{References}

Addison PS (2002) The illustrated wavelet transform handbook. Taylor \& Francis Group, New York

Arens M, Spiegler E (1963) Shock-induced boundary layer separation in overexpanded conical exhaust nozzles. AIAA J 1(3):578-581
Baars WJ, Tinney CE, Ruf JH (2011) Time-frequency analysis of rocket nozzle wall pressures during startup transients. J of Phys: Conference Series 318

Baars WJ, Tinney CE, Ruf JH, Brown AM, McDaniels DM (2012a) Wall pressure unsteadiness and side loads in overexpanded rocket nozzles. AIAA Journal $50(1): 61-73$

Baars WJ, Tinney CE, Wochner MS (2012b) Nonlinear noise propagation from a fully expanded mach 3 jet. In: 50th Aerospace Sciences Meeting and Exhibit, AIAA, Nashville, TN

Blackstock DT (2000) Fundamentals of Physical Acoustics. John Wiley \& Sons, Inc., New York

Cohen L (1989) Time-frequency distributions - a review. Proc of the IEEE 77(7):941-981

Donald BW, Baars WJ, Tinney CE, Ruf JH (2012) Acoustic characterization of sub-scale rocket nozzles. In: 50th Aerospace Sciences Meeting and Exhibit, AIAA, Nashville, TN

Farge M (1992) Wavelet transforms and their application to turbulence. Annu Rev Fluid Mech 24:395-457

Frey M, Hagemann G (2000) Restricted shock separation in rocket nozzles. J of Prop and Power 16(3):478484

Hagemann G, Frey M, Koschel W (2002) Appearance of restricted shock separation in rocket nozzles. J of Prop and Power 18(3):577-584

Lewalle J, Delville J, Bonnet J (2000) Decomposition of mixing layer turbulence into coherent structures and background fluctuations. Flow, Turb and Comb 64:301-328

Nave LH, Coffey GH (1973) Sea level side loads in higharea-ratio rocket engines. In: 9th AIAA Prop. Conf, Las Vegas, NV

Nguyen AT, Deniau H, Girard S, Alziary de Roquefort T (2003) Unsteadiness of flow separation and endeffects regime in a thrust-optimized contour rocket nozzle. Flow, Turb and Comb 71:161-181

Ruf JH, McDaniels DM, Brown AM (2009) Nozzle side load testing and analysis at Marshall Space Flight Center. In: 45th AIAA Joint Prop. Conf, Denver, CO Ruf JH, McDaniels DM, Brown AM (2010) Cold flow test results for nozzle side loads for J-2X and SSME test articles. In: 57th JANNAF Prop. Meet., Colorado Springs, CO

Schmucker RH (1973) Flow processes in overexpanded chemical rocket nozzles. part 2: Side loads due to asymmetric separation. orig. TUM-LRT-TB-10, trans. NASA TM-77395

Verma SB (2009) Shock unsteadiness in a thrust optimized parabolic nozzle. Shock Waves 19(3):193-212

Verma SB, Haidn O (2009) Study of restricted shock separation phenomena in a thrust optimized par- 
abolic nozzle. J of Prop and Power 25(5):1046-57

Verma SB, Stark R, Haidn O (2006) Relation between shock unsteadiness and the origin of side-loads inside a thrust optimized parabolic rocket nozzle. Aerospace Science and Technology 10

Zaman KBMQ, Dahl MD, Bencic TJ, Loh CY (2002) Investigation of a 'transonic resonance' with convergent-divergent nozzles. J Fluid Mech 463 


\section{University Library}

\section{- M M I E E R VA A gateway to Melbourne's research publications}

Minerva Access is the Institutional Repository of The University of Melbourne

Author/s:

Baars, WJ;Tinney, CE

Title:

Transient wall pressures in an overexpanded and large area ratio nozzle

Date:

2013-02-01

Citation:

Baars, W. J. \& Tinney, C. E. (2013). Transient wall pressures in an overexpanded and large area ratio nozzle. EXPERIMENTS IN FLUIDS, 54 (2), https://doi.org/10.1007/ s00348-013-1468-8.

Persistent Link:

http://hdl.handle.net/11343/282578 